\title{
Die Medienberichterstattung zur Vermögens- und Erbschaftsbesteuerung in Deutschland: eine korpuslinguistische Analyse
}

\author{
Hendrik Theine $\cdot$ Andrea Grisold
}

Angenommen: 17. Januar 2022 / Online publiziert: 31. Januar 2022

(C) Der/die Autor(en) 2022

Zusammenfassung Dieser Beitrag untersucht die Berichterstattung zur Vermögensund Erbschaftsbesteuerung im frühen 21. Jahrhundert (2000 bis 2018) in sieben deutschen Tages- und Wochenzeitungen (Welt am Sonntag, Die Zeit, Der Spiegel, Die Welt, Frankfurter Allgemeine Zeitung, Süddeutsche Zeitung und taz).

Der Artikel ist in der Tradition der Politischen Ökonomie der Medien verortet, die die mediale Verbreitung von Diskursen über gesellschaftliche Prozesse als konstitutiv auch für Präferenzen zu wirtschaftspolitischen Fragen ansieht. Medien geben nicht nur Informationen weiter, sondern tragen zur Konstruktion der sozialen Realität bei, indem die Diskurse in die Bevölkerung diffundieren. Internationale empirische Forschungen zur Medienberichterstattung über ökonomische Ungleichheit und die diesbezüglichen Umverteilungsmaßnahmen bilden die Grundlage der hier vorgestellten Studie.

Die Analyse stützt sich auf Methoden der Korpuslinguistik und kommt zu vier zentralen Ergebnissen: erstens zeigt sich im Zeitverlauf, dass die Intensität der Berichterstattung von starken zeitlichen Schwankungen gekennzeichnet ist. Eine systematische Zunahme der Berichterstattung ist dabei aber nicht festzustellen, obwohl die Thematik im Kontext der stark ausgeprägten Vermögensungleichheit steht, deren verstärkte Debatte besteht. Der inhaltliche Fokus liegt zweitens auf der (partei)politischen Ebene, hat eine eindeutig monetäre Dimension, und lässt einen $\mathrm{Zu}$ sammenhang mit gesellschaftspolitischen und wirtschaftlichen Diskursen, die im Kontext der Vermögens- und Erbschaftsbesteuerung stehen (z. B. ökonomische Ungleichheit, Umverteilungswirkungen usw.), auffällig wenig erkennen. Die Analyse einzelner, ausgewählter Zeiträume verstärkt drittens den Befund, dass die (partei)politische Ebene dabei im Vordergrund steht, da Kontroversen im politischen

Hendrik Theine $(\bowtie) \cdot$ Andrea Grisold

Department Volkswirtschaft, Institut für heterodoxe Ökonomie, Wirtschaftsuniversität Wien,

Welthandelsplatz 1, 1020 Wien, Österreich

E-Mail: hendrik.theine@wu.ac.at 
Raum und Reformdebatten die dominanten Anlässe der Berichterstattung darstellen. Viertens ergibt die Analyse der einzelnen Zeitungen, dass die Ausrichtung der Berichterstattung in allen Zeitungen durchaus ähnlich ausgerichtet ist; einige Differenzen ergeben sich hinsichtlich unternehmensbezogener Aspekte (Die Welt, Welt am Sonntag und FAZ), Details zur Ausgestaltung der Steuern (Süddeutsche Zeitung) sowie dem Zusammenhang zwischen Erbschafts- und Vermögenssteuern mit Krisenentwicklungen und dem Klimawandel (taz).

Schlüsselwörter Politische Ökonomie der Medien · Vermögensbesteuerung · Medienberichterstattung $\cdot$ Korpuslinguistik $\cdot$ Umverteilungsmaßnahmen

\section{The media coverage of wealth and inheritance taxation in Germany: a corpus linguistic analysis}

Abstract This paper examines the coverage of wealth and inheritance taxation in the early 21 st century (2000 to 2018) in seven German daily and weekly newspapers (Welt am Sonntag, Die Zeit, Der Spiegel, Die Welt, Frankfurter Allgemeine Zeitung, Süddeutsche Zeitung and taz).

Rooted in the tradition of Political Economy of the Media, discourses in the media constitute preferences, not to the least for public policies. Media are not neutral information provider, but active in constructing social reality, influencing the public via the discourses told. International empirical studies analysing the media coverage on economic inequality and redistribution policies serve as basis for the present study.

Building on corpus linguistics, this paper shows that wealth and inheritance taxation is a rather infrequent topic over the entire period, with the exception of a few months of intense, increased reporting. Content-wise, the coverage focusses on the political (party) level, has a clear monetary dimension and indicates an-albeit rather weak-connection to socio-political and economic debates. The analysis of selected time periods reinforces the evidence that the political (party) level dominates, as controversies in the political sphere and debates on reforming the inheritance tax are the central occasions for increased coverage. Ultimately, the analyses of the single newspapers confirm the reporting between the different newspapers as quite similar; minor differences arise with regard to company-related aspects (Die Welt, Welt am Sonntag and FAZ), a more detailed coverage of the tax design (Süddeutsche Zeitung), and a connection of inheritance and wealth taxes with crisis developments and climate change (taz).

Keywords Political economy of the media - Wealth taxation - Media coverage Corpus linguistics $\cdot$ Redistribution policies

\section{Einleitung}

Seit den 1980er-Jahren hat die Vermögensungleichheit in vielen Ländern zugenommen, so die Befunde einer steigenden Zahl empirischer Untersuchungen (Piketty und 
Zucman 2015; Piketty 2014). Für jene Länder, die gute Langzeitdaten vorweisen, kann weiters belegt werden, dass die generationenübergreifende Übertragung von Vermögen - in Form von Erbschaften und Schenkungen - einen wesentlichen Bestimmungsfaktor der anhaltenden Vermögensungleichheit darstellt (Adermon et al. 2018; Piketty 2011; Roine und Waldestrom 2009). Neben der empirischen Analyse der Vermögensverteilung sind in den letzten Jahren vermehrt Forschungsergebnisse zu den Auswirkungen steigender Vermögensungleichheit auf Gesellschaft und Ökonomie zu verzeichnen. So verdichten sich auch die Befunde, dass eine hohe Konzentration von Privatvermögen in engem Zusammenhang mit ökonomischer und politischer Macht steht. Damit kommt der Verteilung von Vermögen auch eine zentrale Rolle im Funktionieren demokratischer Institutionen zu (Rehm und Schnetzer 2015; Elsässer et al. 2017; Theine 2020; Theine und Taschwer 2021).

Einen zentralen Treiber für zunehmende Vermögensungleichheit stellen sinkende Steuersätze auf Vermögen und Erbschaften dar (Scheve und Stasavage 2016; Piketty und Zucman 2015; Beckert 2013). So betrug nach dem Zweiten Weltkrieg der Spitzensteuersatz auf Erbschaften in Industrieländern noch im Schnitt 30-40\%. Diese wurden ab den 1980er-Jahren gesenkt oder ganz abgeschafft. Ähnlich verhält es sich bei der Vermögensteuer: Während vor einigen Jahrzehnten noch die Hälfte der OECD-Länder Vermögen besteuerten, sind dies mittlerweile nur noch drei Länder: die Schweiz, Norwegen und Spanien (Drometer et al. 2018).

Während die oben skizzierten Entwicklungen mittlerweile einen empirisch gut abgesicherten Forschungsstand aufweisen, blieb die Rolle der Massenmedien in der Vermittlung von Vermögensungleichheit und Umverteilungsmaßnahmen bisher weitgehend unbeachtet (Grisold und Theine 2017, 2020). Das mutet, angesichts der Bedeutung des Themas und der zentralen Rolle der Medien für öffentliche Wahrnehmung, durchaus überraschend. Hier setzt die vorliegende Untersuchung mit Folgender Forschungsfrage an: Welche Diskurse werden von der Vermögens- und Erbschaftsbesteuerung in der massenmedialen öffentlichen Diskussion vermittelt und befördert?

Mittels korpuslinguistischer Methoden wird die Medienberichterstattung zur Vermögens- und Erbschaftsbesteuerung im frühen 21. Jahrhundert (2000 bis 2018) in ausgewählten deutschen Printmedien hinsichtlich des Stellenwert des Themenkomplexes sowie gemäß der textlichen Ausrichtung untersucht. ${ }^{1}$ Dieser Beitrag kommt zu vier zentralen Ergebnissen: erstens zeigt sich im Zeitverlauf, dass die Intensität der Berichterstattung von starken zeitlichen Schwankungen gekennzeichnet, eine systematische Zunahme der Berichterstattung aber nicht festzustellen ist, obwohl die Thematik im Kontext der stark ausgeprägten Vermögensungleichheit steht und damit verstärkte Debatte besteht. Der inhaltliche Fokus liegt zweitens auf der (partei)politischen Ebene, hat eine eindeutig monetäre Dimension, und lässt einen Zusammenhang mit gesellschaftspolitischen und wirtschaftlichen Debatten, die im Kontext der Vermögens- und Erbschaftsbesteuerung stehen (z. B. ökonomische Ungleichheit, Umverteilungswirkungen usw.), auffällig wenig erkennen. Die Analyse einzelner, ausgewählter Zeiträume verstärkt drittens den Befund, dass die (par-

\footnotetext{
${ }^{1}$ Siehe auch Theine und Grisold (2020) sowie Theine (2021) für ähnlich gelagerte Analysen zu dieser Thematik.
} 
tei)politische Ebene im Vordergrund steht, da Kontroversen im politischen Raum und Reformdebatten die dominanten Anlässe der Berichterstattung darstellen. Viertens ergibt die Analyse der einzelnen Zeitungen, dass die Ausrichtung der Berichterstattung in allen Zeitungen durchaus ähnlich ausgerichtet ist; einige Differenzen ergeben sich hinsichtlich unternehmensbezogener Aspekte (Die Welt, Welt am Sonntag und Frankfurter Allgemeine Zeitung), Details zur Ausgestaltung der Steuern (Süddeutsche Zeitung) sowie dem Zusammenhang zwischen Erbschafts- und Vermögenssteuern mit Krisenentwicklungen und dem Klimawandel (taz).

Der Beitrag ist wie folgt strukturiert: $\mathrm{Zu}$ Beginn wird die Besteuerung von Vermögen und Erbschaften in Deutschland diskutiert (Abschn. 2). Anschließend wird die relevante Literatur im Bereich Medien und Ungleichheitsforschung diskutiert (Abschn. 3) und das Forschungsdesign der hier präsentierten Studie erläutert (Abschn. 4). Die Darstellung der empirischen Ergebnisse wird in Abschn. 5 vorgenommen. Eine zusammenfassende Einschätzung erfolgt in Abschn. 6.

\section{Hintergrund: Vermögens- und Erbschaftssteuern in Deutschland}

In Bezug auf die Vermögensverteilung ist Deutschland eines der ungleichsten Länder im Euroraum (Bach et al. 2019; Leitner 2016). Die obersten zehn Prozent besitzen rund $65 \%$, die obersten ein Prozent besitzen sogar rund $35 \%$ des gesamten privaten Vermögens. Im Gegensatz dazu besitzen die ,unteren“ $50 \%$ ca. drei Prozent des gesamten Vermögens (Werte sind für das Jahre 2014 angegeben). Der Gini-Koeffizient, ein statistisches Maß für die Ungleichheit einer Verteilung, liegt damit bei 0,79. Zum Vergleich: In Frankreich besitzen die obersten ein Prozent rund $25 \%$ des gesamten privaten Vermögens, in Spanien sind es knapp unter 20\% (Bach et al. 2019).

Diese Ungleichheit in der Vermögensverteilung weist eine hohe Kontinuität auf. Haushalte mit hohem Vermögen haben im Laufe der Zeit ihre Position innerhalb der Vermögensverteilung kaum verändert. Gleichzeitig ist es sehr unwahrscheinlich, dass Haushalte mit wenig oder gar keinem Vermögen ihren Vermögensbestand deutlich erhöhen können (Bach et al. 2019; Grabka 2014). Ein wesentlicher Grund für die anhaltende Vermögensungleichheit ist die generationsübergreifende Übertragung in Form von Schenkungen und Erbschaften, in Deutschland werden etwa 25 bis $40 \%$ des Gesamtvermögens vererbt (Fessler und Schürz 2018; Leitner 2016; Piketty 2014).

Auch die Besteuerung von Vermögen und Erbschaften spielt eine wichtige Rolle für die Vermögensungleichheit. Ein Blick auf die langfristige Entwicklung der Erbschafts- und Vermögensbesteuerung zeigt, dass beide Mitte des 20. Jahrhunderts vergleichsweise stark besteuert wurden; in der zweiten Hälfte des letzten Jahrhunderts wurden die Steuersätze dann schrittweise reduziert (Houben und Maiterth 2011; Wieland 2003; Bach 1997). Im Jahr 1995 entschied das Bundesverfassungsgericht, dass die Vermögenssteuer in der damals bestehenden Form verfassungswidrig ist, da unterschiedliche Vermögensarten unterschiedlich besteuert wurden. Da die zweijährige Frist zur Reform der Vermögenssteuer nicht genutzt wurde, ist diese seit 
1997 in Deutschland de facto ausgesetzt (van Kommer und Kosters 2013; Bach und Beznoska 2012).

Im 21. Jahrhundert - dem in diesem Beitrag gewählten Betrachtungszeitraum - ist die Erbschaftsbesteuerung zweimal (2006 und 2014) durch das Bundesverfassungsgericht als in Teilen verfassungswidrig eingestuft worden. Im Mittelpunkt beider Gerichtsurteile standen die weitreichenden Steuerbefreiungen für die Unternehmensvererbung und das damit einhergehende Versagen des Gesetzgebers, unterschiedliche Vermögensarten gleich zu behandeln. In beiden Fällen erhielt der Gesetzgeber etwa zwei Jahre Zeit, um das Steuergesetz zu reformieren, was dann auch 2009 und 2016 erfolgte. In beiden Reformprozessen wurde die vom Verfassungsgericht abgelehnte Steuerbegünstigung von Unternehmensvermögen geändert und reformiert, gleichzeitig wurden aber neue Befreiungen ähnlicher Art eingeführt (Bach 2016; Maiterth 2013; Houben und Maiterth 2011).

Die neu eingeführten Regelungen haben nur geringe Veränderungen hinsichtlich der Steuerbefreiung von Erbschaften mit sich gebracht. Zwischen 2009 und 2015 sind trotz der eingeführten Steuerreform nach wie vor über $150 \mathrm{Mrd}$. $€$ an Firmenvermögen steuerfrei vererbt worden. In den Jahren 2011 bis 2014 waren Übertragungen ab fünf Millionen Euro im Durchschnitt zu mehr als der Hälfte steuerbefreit, Übertragungen ab 20Mio. $€$ waren in diesem Zeitraum im Schnitt sogar zu $93 \%$ steuerbefreit $^{2}$ (Bach 2016; Bach und Mertz 2016). Auch die Reform von 2016 hat an dieser Situation nichts grundsätzlich verändert. Zwar wurden die Vererbungsregeln für Betriebsvermögen weiter verkompliziert, um den Anforderungen des Bundesverfassungsgerichts zu entsprechen. Die grundlegende Tendenz der weitreichenden Steuerbefreiung von Betriebsvermögen bleibt aber bestehen (Franke et al. 2016; Scholz und Truger 2016).

Die niedrige Besteuerung von Erbschaften und Schenkungen führt dazu, dass die Steuereinnahmen aus der Erbschafts- und Schenkungssteuer in Deutschland zwischen 2000 und 2018 nur vier bis acht Milliarden Euro pro Jahr ausmachten. Im Vergleich dazu sind die Steuereinnahmen aus der Umsatz- und Lohnsteuer um ein Vielfaches höher (Umsatzsteuer 2000 bis 2018: 104 Mrd. $€$ bis 174 Mrd. $€$; Lohnsteuer: 123 Mrd. $€$ bis 208 Mrd. $€$ ) (Destatis 2018). Die anhaltend niedrige Besteuerung von Erbschaften und die Aussetzung der Vermögenssteuer seit 1997 führte - gemeinsam mit weiteren Steuerreformen der vergangenen 20 Jahre $^{3}-$ zu einer eindeutigen Verschiebung der Steuerbeiträge zulasten der unteren Einkommen. Die unteren $70 \%$ zahlen 2015 anteilig mehr Steuern als noch im Jahr 1998, während die obersten $30 \%$ weniger zum Staatshaushalt beitragen (Bach et al. 2019; Sachverständigenrat 2019).

\footnotetext{
2 Besonders bemerkenswert ist in dieser Phase, dass im Rahmen von sogenannten „Vorzieheffekten“ die Übertragung von Vermögen zu Lebzeiten aufgrund der steuerlichen Vorteile signifikant angestiegen ist, d.h., dass eine beachtliche Zahl an Minderjährigen in diesem Zeitraum zu Milliardär*innen geworden ist (Bach 2016; Bach und Mertz 2016).

3 Neben der Aussetzung der Vermögenssteuer und der Reform der Erbschaftssteuer ist hier noch die Reduktion des Einkommenssteuerhöchstsatzes, die Unternehmenssteuerreform sowie die Absenkung der Kapitalertragssteuer zu nennen. Insgesamt hat damit die Progressions- und Umverteilungswirkung des Steuersystems zwischen 1998 und 2015 abgenommen (Bach et al. 2019).
} 
Die Wiedereinführung der Vermögensteuer und die Reform der Erbschaftssteuer würden hier ansetzen, um vermögende Gesellschaftsgruppen wieder stärker an der Finanzierung staatlicher Aufgaben zu beteiligen. Bach und Thiemann (2016) berechnen beispielsweise, dass schon eine Vermögenssteuer von nur 1\% (mit hohen Freibeträgen bis zu einer Millionen Euro) zu zusätzlichen jährlichen Steuereinnahmen von 10 bis $20 \mathrm{Mrd}$. $€$ führen würde. Steuerpflichtig wären dabei nur ca. 150.000 Personen, das entspricht den reichsten 0,2 bis 0,5\% der Bevölkerung in Deutschland. Ähnliches gilt für die Erbschaftssteuer. Schon bei einer moderaten Reform, bei der die weitreichenden Steuerbegünstigungen von Unternehmensvermögen eingeschränkt würden, können - bei Steuersätzen von $15 \%$ und einem Freibetrag bis zu $500.000 €$ pro Person - Steuereinnahmen von 15 bis 20 Mrd. $€$ erzielt werden (Bach 2016).

\section{Medien und Ungleichheitsforschung}

Vor dem Hintergrund der im Abschn. 2 thematisierten Problematik ist es naheliegend, zu hinterfragen, wie dieses Problem medial vermittelt wird. Dies erfolgte mit der vorliegenden Untersuchung, die in der Forschungstradition der „Politischen Ökonomie der Medien“ steht. Dieser kritische Ansatz zeichnet sich durch das zentrale Bestimmungsmerkmal aus, kapitalistische soziale Beziehungen als machtvermittelt zu verstehen. Die Verteilung und der Konsum von gesellschaftlichen Ressourcen sind damit abhängig von Auseinandersetzungen zwischen unterschiedlichen sozialen Klassen, die in einer Gesellschaft um Vorherrschaft ringen (Mosco 2009). In solch einer Auseinandersetzung spielen die Medien eine wesentliche Rolle, da sie den Raum bilden, in dem Machtverhältnisse zwischen konkurrierenden sozialen und politischen Akteuren entschieden werden (Castells 2009).

Medien sind in dieser Sichtweise Großteils gewinnorientierte Unternehmen, haben aber noch eine weitere Funktion, die im vorliegenden Artikel auch im Vordergrund steht: die der Herstellung und Verbreitung von Diskursen über gesellschaftliche Prozesse, welche in die Bevölkerung diffundieren (Mosco 2009; Grisold 2004). Hier ist hervorzuheben, dass Medien nicht nur Informationen verbreiten, sondern eben auch zur Konstruktion der sozialen Realität beitragen. Die Rolle der Medien ist damit allumfassend in dem Sinne, dass die uns bekannte Form der gesellschaftlichen Wirklichkeit ohne diese gar nicht denkbar wäre (Grisold 2009, 2004). Mit anderen Worten: Das Weltbild der Menschen wird mit Diskursen, Symbolen und Bildern über die Medien geformt und hier insbesondere das konzeptuelle Wissen, von dem das jeweilige eigene Weltverständnis entscheidend abhängt (Schulz 1989). Dies gilt auch für die vorliegende Thematik: Die Medienberichterstattung beeinflusst die öffentliche Meinung sowie die individuellen Einstellungen zur ökonomischen Ungleichheit und damit zusammenhängend zu Umverteilungsmaßnahmen (Epp und Jennings 2020; Grisold und Theine 2020).

Methodisch schließt der vorliegende Beitrag an empirische Forschungen zur Medienberichterstattung über ökonomische Ungleichheit und die diesbezüglichen Umverteilungsmaßnahmen an (für eine ausführliche Besprechung der bestehenden Literatur siehe Grisold und Theine 2017, 2020). Quantitative Untersuchungen zur 
Medienberichterstattung über ökonomische Ungleichheit konstatieren, dass die Berichterstattung zwischen 1945 und 2015 in großen deutschen Zeitungen tendenziell zugenommen hat (Schröder und Vietze 2015; Petring 2016). Insbesondere im letzten Jahrzehnt ist eine Intensivierung der Berichterstattung zum Thema Ungleichheit zu verzeichnen; die Intensität der Berichterstattung geht dabei überwiegend mit der politischen Ausrichtung der untersuchten Medien d' accord (Schröder und Vietze 2015). Die Quantität der Berichterstattung zu ökonomischer Ungleichheit sagt allerdings noch wenig über die Vermittlung von Inhalten aus. Epp und Jennings (2020) zeigen, dass im Rahmen der US-amerikanischen Berichterstattung zur ökonomischen Ungleichheit die Charakterisierung von Armut als persönliches Versagen im Zeitverlauf stark zugenommen hat. Sie können auch nachweisen, dass diese Entwicklung mit einer sinkenden Akzeptanz von wohlfahrtsstaatlichen Maßnahmen einhergeht.

Qualitative Analysen zeigen weiterhin, dass die politische Orientierung der Medien eine starke Rolle in der inhaltlichen Ausrichtung spielt, hat doch die Berichterstattung über Ungleichheit in konservativen Zeitungen nur geringfügig zugenommen, während progressiver ausgerichtete Zeitungen ihre Berichterstattung intensiviert haben (Bank 2017) sowie ökonomische Ungleichheit oft im Rahmen des dominanten Leistungsnarrativs interpretiert wird (Smith Ochoa 2020).

Jene wissenschaftlichen Studien, welche die Medienberichterstattung zu Umverteilungsmaßnahmen (von Vermögen und Einkommen) untersuchen, konstatieren, dass diese in der Berichterstattung eher ablehnend behandelt werden, die positiven Implikationen der Umverteilungspolitik, d. h. die Reduktion von Ungleichheit, vielerorts unterrepräsentiert bleiben (siehe u. a. Lichtenstein et al. 2016). Wird die Piketty-Debatte 2014/15 $5^{4}$ herangezogen, so ergibt eine Analyse der Berichterstattung in jeweils drei Tages- und Wochenzeitungen in Irland, Großbritannien, Deutschland und Österreich, dass Umverteilungspolitiken in geringerem Maße angesprochen werden als das Thema der ökonomischen Ungleichheit an sich, und diese Politiken außerdem als wesentlich umstrittenerer definiert werden (Grisold und Preston 2020). Wenn Themen wie höhere Vermögens- und Einkommenssteuern Erwähnung finden, so werden sie eher flüchtig angesprochen und nicht weiter detailliert behandelt. Darüber hinaus lassen sich linguistische Zuspitzungen und aggressiv ablehnende Formulierungen feststellen, welche die Gefährlichkeit, Unmöglichkeit und Ineffizienz einer höheren Besteuerung vermitteln sollen. So werden zum Beispiel , die Reichen' gern als Opfer ohne Handlungskompetenz portraitiert, welche ein ,aggressiver', ,überwältigender' Staat den Risiken durch Kapitalentnahme und daher der Gefahr des Konkurses aussetzt (Rieder und Theine 2019; Theine und Rieder 2019; Grisold und Theine 2018).

\footnotetext{
${ }^{4}$ Im Jahr 2014 wurden sowohl die englischsprachige als auch die deutschsprachige Übersetzung des Buchs „Capital in the 21st Century“ (Kapital im 21. Jahrhundert) von Thomas Piketty veröffentlicht. Das Buch wurde weltweit medial stark rezipiert und führte zu einer öffentlich breit geführten Debatte um ökonomische Ungleichheit und Umverteilung von Einkommen sowie Vermögen (Bank 2015; Theine und Grabner 2020).
} 


\section{Forschungsdesign}

Für die Analyse der Berichterstattung zur Vermögens- und Erbschaftsbesteuerung im frühen 21. Jahrhundert wurden sieben deutsche Tages- und Wochenzeitungen ausgewählt: Welt am Sonntag, Die ZEIT, Der Spiegel ${ }^{5}$, Die Welt, Frankfurter Allgemeine Zeitung (FAZ), Süddeutsche Zeitung (SZ) und taz. Die Auswahl der Zeitungen ergibt sich aus ihrer Stellung als einflussreiche und vielgelesene Qualitätszeitungen in Deutschland. Einige von ihnen werden in die Rubrik der „Leitmedien“ gereiht; darunter werden solche Zeitungen verstanden, die soziale, politische und wirtschaftliche Debatten beeinflussen und prägen (Röper 2018; Weischenberg et al. 2005).

Der deutsche Zeitungsmarkt weißt recht hohe Konzentrationstendenzen auf (Ferschli et al. 2019), was das Risiko einer geringen oder negativen Berichterstattung über ökonomische Ungleichheit und Umverteilungsmaßnahmen beinhaltet (Neimanns 2021). Die Eigentumsverhältnisse betreffend, sind fünf der sieben Zeitungen Teil von großen Medienunternehmen; dazu gehören mehrgenerationale Familienunternehmen wie Gruner+Jahr, Verlagsgruppe von Holtzbrinck oder die Axel Springer $S E$. Insbesondere bei diesen Familienunternehmen wird nicht nur das Eigentum an den Unternehmen, sondern damit auch erhebliches Vermögen über Generationen weitergegeben (Ferschli et al. 2019). Die genannten Unternehmen dürften von der Eigentümerseite her also ein ausgeprägtes Interesse an einer ablehnenden Haltung zur Vermögens- und Erbschaftsbesteuerung haben. ${ }^{6}$ Zwei Zeitungen unterscheiden sich jedoch deutlich hinsichtlich der Besitzverhältnisse: taz und FAZ sind im Eigentum jeweils einer Genossenschaft bzw. einer gemeinnützigen Stiftung (Bergmann und Novy 2012; Groll 2012).

Die politische Ausrichtung und somit auch die redaktionelle Haltung der sieben Zeitungen weisen ebenfalls Unterschiede auf. Unter Beachtung der zwangsläufigen Vereinfachungen, die mit solch allgemeinen Charakterisierungen einhergehen, kann Welt am Sonntag und Die Welt als bürgerlich-konservativ ausgerichtet bezeichnet werden, mit ausgeprägten marktliberaler Haltung zu wirtschaftspolitischen Themen (Sasse 2012; Pointner 2010). Die ZEIT genießt den Ruf einer Wochenzeitung mit hochwertigem Journalismus, dessen redaktionelle Haltung als linksliberal bezeichnet wird. Der Spiegel, war in früheren Jahrzehnten als progressiv ausgerichtete Zeitschrift bekannt, tendierte aber in der jüngeren Verlagsgeschichte mehr und mehr zu (neo-)liberalen Positionen (Wolter 2016; Burkhardt 2012; Augstein 2005; Gehrs 2005). Gegründet von einer Gruppe einflussreicher deutscher Industrieller, schwankt der Ton der $F A Z$ seit ihren Anfängen zwischen liberalen und konservativen Positionen (Burkhardt 2012; Schulz 1986). Die aktuelle Forschung zeigt, dass die FAZ insbesondere bei wirtschaftspolitischen Themen eher marktliberal ausgerichtet ist

\footnotetext{
${ }^{5}$ Nachfolgend wird Der Spiegel, da er als Leitmedium gilt, in der Analyse mitberücksichtigt, obwohl er nicht als Wochenzeitung gilt, sondern als wöchentliches Nachrichtenmagazin. Der Einfachheit halber wird er nachstehend im Text unter den wöchentlichen Publikationen nicht jedes Mal gesondert als Magazin aufgeführt.

${ }^{6}$ Um die redaktionelle Eigenständigkeit trotz solch spezifischer Eigentümerinteressen zu wahren, haben sich historisch unterschiedliche Regelungen entwickelt, die erstere sicherstellen sollen. Dass dies nicht immer gelingt, wird in konzeptionellen und empirischen Studien immer wieder betont (siehe z. B. Herman und Chomsky 2002; Benson 2016, 2019).
} 
Tab. 1 Übersicht über die ausgewählten Zeitungen und die Anzahl der untersuchten Zeitungsartikel

\begin{tabular}{|c|c|c|c|c|}
\hline Zeitung & $\begin{array}{l}\text { Erscheinungsart } \\
\text { (Zeitungstyp) }\end{array}$ & EigentümerInnen & $\begin{array}{l}\text { Anzahl der } \\
\text { untersuchten } \\
\text { Artikel }\end{array}$ & $\begin{array}{l}\text { Anteil an allen } \\
\text { untersuchten } \\
\text { Artikeln }(\%)\end{array}$ \\
\hline $\begin{array}{l}\text { Welt am } \\
\text { Sonntag }\end{array}$ & Wöchentlich & Axel Springer SE & 703 & 7,2 \\
\hline Die ZEIT & $\begin{array}{l}\text { Wöchentlich } \\
\text { (Leitmedium) }\end{array}$ & $\begin{array}{l}\text { Verlagsgruppe von Holtz- } \\
\text { brinck }\end{array}$ & 644 & 6,6 \\
\hline Der Spiegel & $\begin{array}{l}\text { Wöchentlich } \\
\text { (Leitmedium) }\end{array}$ & $\begin{array}{l}\text { Gruner+Jahr, Spiegel- } \\
\text { Mitarbeiter KG, Rudolf } \\
\text { Augstein Erben }\end{array}$ & 431 & 4,4 \\
\hline Die Welt & Täglich & Axel Springer SE & 2332 & 24 \\
\hline $\begin{array}{l}\text { Frankfurter } \\
\text { Allgemeine } \\
\text { Zeitung }\end{array}$ & $\begin{array}{l}\text { Täglich (Leit- } \\
\text { medium) }\end{array}$ & Fazit-Stiftung & 1077 & 11,1 \\
\hline $\begin{array}{l}\text { Süddeutsche } \\
\text { Zeitung }\end{array}$ & $\begin{array}{l}\text { Täglich (Leit- } \\
\text { medium) }\end{array}$ & $\begin{array}{l}\text { Südwestdeutsche Medien } \\
\text { Holding }\end{array}$ & 2944 & 30,3 \\
\hline Taz & Täglich & $\begin{array}{l}\text { Tageszeitung Verlagsge- } \\
\text { nossenschaft eG }\end{array}$ & 1580 & 16,3 \\
\hline Summe & & & 9711 & 100 \\
\hline
\end{tabular}

Quelle: Ferschli et al. (2019), kek (2019), Bergmann und Novy (2012), Groll (2012) eigene Berechnungen

(Wolter 2016; Pointner 2010; Volkmann 2006). Der SZ wird eher eine linksliberale Haltung zugeschrieben, obwohl Medienwissenschaftler wie Wolter (2016) zeigen, dass in der wirtschaftspolitischen Berichterstattung auch neoliberale Argumente und Einschätzungen wirkmächtig sind. Mit der taz, gegründet als selbstorganisierte, basisdemokratische Zeitung, ist schließlich ein Presseorgan vertreten, das der politisch linken, grünalternativen Öffentlichkeit zugerechnet wird (Petring 2016; Groll 2012). Obwohl sich die politischen Ausrichtungen und somit auch die redaktionellen Haltungen der untersuchten Medien durchaus unterscheiden, zeigen bisherige Analysen gleichwohl auch medienübergreifende, diskursive Muster und Gemeinsamkeiten, insbesondere im Bereich der wenig ausgeprägten Kritik an marktliberalen Ordnungen (Krüger et al. 2021; Nullmeier et al. 2014).

Tab. 1 beinhaltet die betrachteten Zeitungen, deren Besitzverhältnisse und die Anzahl der Artikel, die sich im Untersuchungszeitraum mit dem Thema der Vermögens- und Erbschaftsbesteuerung auseinandergesetzt haben. Für einen relativen Vergleich, welche Wichtigkeit diesem Thema in der jeweiligen Zeitung beigemessen wird, sind in der letzten Spalte die Anteile der relevanten Artikel je Zeitung in Relation zu allen untersuchten Artikeln aufgeführt. $\mathrm{Zu}$ beachten ist, dass natürlich nur die Tages- und Wochenzeitungen jeweils sinnvoll untereinander verglichen werden können. 
Die Zeitungsartikel zur Vermögens- und Erbschaftsbesteuerung wurden aus mehreren Datenbanken (Lexis Nexis, factiva und WISO) mit geeigneten Stichworten ${ }^{7}$ bezogen. Der letztendliche Korpus umfasst 9711 Artikeln. In den drei Wochenzeitungen sind im gesamten Zeitraum zwischen 500 und 700 Artikel erschienen; in den vier Tageszeitungen schwankt die Anzahl im gesamten Zeitraum zwischen 1000 und 3000 Artikel.

Die Intensität der Berichterstattung ist in den sieben Zeitungen dabei durchaus unterschiedlich. In den beiden Zeitungen Die Zeit und Der Spiegel befassen sich 0,4\% bzw. 0,34\% der gesamten, in diesen Zeitungen veröffentlichten, Artikeln mit der Erbschafts- und Vermögensbesteuerung. Im Vergleich dazu sind es in Die Welt, Welt am Sonntag und taz um die 0,2\% der Artikel. Die FAZ berichtet hingegen mit nur $0,08 \%$ am wenigsten über Erbschafts- und Vermögenssteuern. Die relative Anzahl der Artikel deutet insgesamt auf ein eher geringes Interesse der Zeitungen an der Thematik der Vermögens- und Erbschaftsbesteuerung hin; insbesondere, wenn man die gesellschaftliche Bedeutung des Themas (siehe Abschn. 2 „Hintergrund: Vermögens- und Erbschaftssteuern in Deutschland“) als Maßstab nimmt.

Die Berichterstattung zu Vermögens- und Erbschaftssteuern wird in diesem Beitrag mit Methoden der Korpuslinguistik analysiert (Subtirelu und Baker 2017; Lemke und Wiedemann 2016). Der gängigen Praxis der quantitativen Textanalyse folgend, wurde in einem ersten Schritt Stoppwörter (z.B. „das“, „und“, „oder“), Anführungszeichen und Satzzeichen entfernt. Außerdem wurden die unterschiedlichen Schreibweisen der Wörter Erbschaftssteuer und Vermögenssteuer für die Analyse vereinheitlicht. Mit Hilfe des Sprachmodells von UDpipe wurden die in den Zeitungsartikeln vorkommenden Wörter zu ihren jeweiligen Wortklassen zugeordnet (Straka 2018). Diese Zuordnung hat den Vorteil, dass spezifische Wortklassen (z. B. Nomen, Verben und Adjektive) für die weitere Analyse berücksichtigt werden können, während andere, weniger relevante Wortklassen (z. B. Pronomen, Präpositionen und Konjunktionen) systematisch ausgeschlossen werden.

Zur Ermittlung der Häufigkeit von Wörtern und Wortkombinationen wurden Wort- und Bigramm-Häufigkeitslisten ${ }^{8}$ berechnet. Solch häufig verwendete Wörter und Wortkombinationen sind ein zentrales Instrument, um die generelle Ausrichtung der Berichterstattung zu ermitteln und einen allgemeinen Überblick über den Korpus zu erlangen (Silge und Robinson 2017). Für eine vertiefende Analyse ausgewählter Stichworte wurden zum einen die im gleichen Satz befindlichen Nomen, Verben und Adjektive analysiert. Zum anderen wurden die Stichworte mittels KWICKonkordanzen einer eingehenden Analyse unterzogen. KWIC (,keywords-in-context")-Konkordanzen ermöglichen es zu untersuchen, welche Art von Worten ein bestimmtes Schlüsselwort umgeben (Haider 2017; Mautner 2015). Letztlich wurde die inverse Dokumenthäufigkeit von Wörtern berechnet, um festzustellen, welche Worte in einzelnen Zeitungen einzigartig sind und somit nicht oder nur sehr selten

\footnotetext{
7 Mit den folgenden Stichworten wurden die Zeitungsartikel ausgewählt: Vermögenssteuer, Vermögensteuer, Vermögensbesteuerung, Vermögenbesteuerung, Vermögensabgabe, Vermögenabgabe, Reichensteuer, Erbschaftssteuer, Erbschaftsteuer, Erbschaftsbesteuerung, Erbschaftbesteuerung, Erbschaftsabgabe, Erbschaftabgabe, Reichenabgabe, Reichenbesteuerung.

8 Bigramme sind Sequenzen von zwei benachbarten Wörtern.
} 
in anderen Zeitungen vorkommen. Die inverse Dokumenthäufigkeit misst dabei, wie wichtig ein bestimmtes Wort in einen ausgewählten Teilkorpus (hier: die einzelnen Zeitungen) im Vergleich zur Gesamtmenge der betrachteten Dokumente ist (Silge und Robinson 2017).
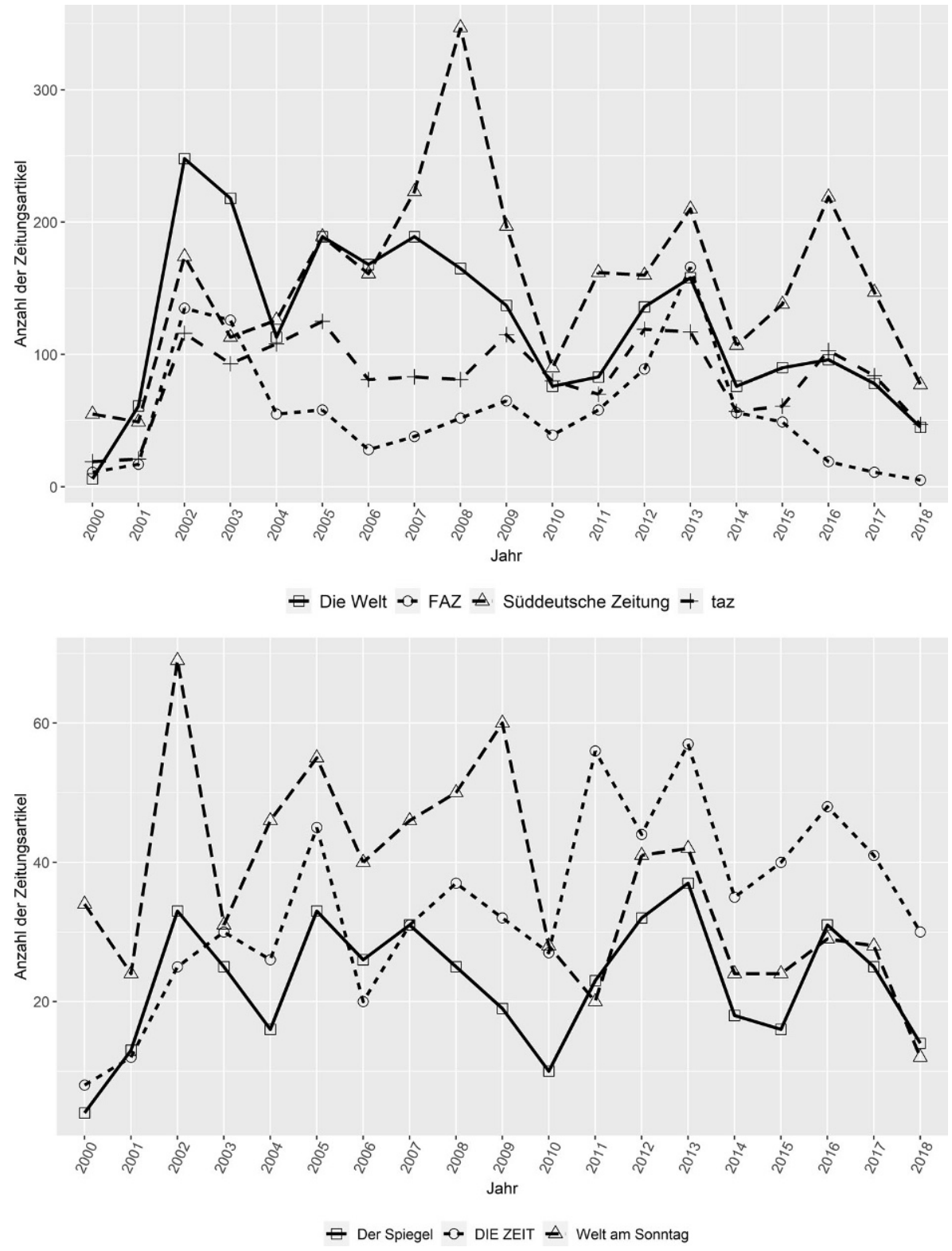

Abb. 1 Anzahl der Artikel zur Vermögens- und Erbschaftsbesteuerung, pro Zeitung im Zeitverlauf 2000 bis 2018 (oben: Tageszeitungen; unten: Wochenzeitungen) 


\section{Ergebnisse: Intensität, thematischer Schwerpunkt und Anlässe der Berichterstattung}

In diesem Abschnitt werden die Ergebnisse hinsichtlich der Intensität der Berichterstattung und der diskursiven Ausrichtung analysiert, sowie zeitungsspezifische und zeitliche Unterschiede dargestellt und diskutiert.

\subsection{Die Intensität der Berichterstattung}

Die Intensität der Berichterstattung ist von starken zeitlichen Schwankungen gekennzeichnet. So zum Beispiel im Jahr 2002: während bis Mitte des Jahres noch vergleichsweise wenig (zwischen 15 und 30) Artikel pro Monat veröffentlicht worden sind, nimmt die Anzahl ab September (70 Artikel) bis Dezember (287 Artikel) rasch zu. Ähnliche sprunghafte Anstiege in der Berichterstattung lassen sich auch in weiteren Zeitperioden identifizieren; auf diese Perioden wird im nächsten Kapitel vertieft eingegangen.

Die Anzahl der Artikel pro Zeitung im zeitlichen Verlauf (Abb. 1) zeichnet ein ähnliches Bild mit relativ starken Schwankungen. Einige Phasen intensiverer Berichterstattung finden in mehreren Zeitungen annähernd parallel statt. So steigt die Anzahl der Artikel in den Jahren 2002, 2005, 2013 sowie 2016 in fast allen Zeitungen relativ synchron an (siehe genauer im nächsten Abschnitt). In Gegensatz dazu ist die intensivere Berichterstattung im Jahr 2008 allein von der $S Z$ getragen.

Eine systematische Zunahme der Berichterstattung über den gesamten Untersuchungszeitraum ist insgesamt nicht festzustellen. In beinahe allen Zeitungen ist die absolute Anzahl der Artikel zur Erbschafts- und Vermögensbesteuerung in den Jahren 2000 und 2001 etwa gleich hoch wie in 2017 und 2018. Hinsichtlich der einzelnen Zeitungen ist bei Die Welt und Welt am Sonntag ab 2002 sogar eine konstante Abnahme der Berichterstattung zu verzeichnen; bei Die Zeit lässt sich hingegen eine leichte Zunahme feststellen.

\subsection{Der thematische Schwerpunkt der Berichterstattung}

Die diskursive Ausrichtung der Berichterstattung zu Erbschafts- und Vermögenssteuern wird in einem ersten Schritt mittels der häufigsten Schlagwörter und Wortkombinationen analysiert, bevor anschließend näher auf die textuelle Einbettung ausgewählter der Schlüsselwörter eingegangen wird.

Tab. 2 zeigt die häufigsten Wörter und Wortkombinationen in allen Zeitungen im gesamten Untersuchungszeitraum. Die drei Spalten enthalten Informationen über das Wort, dessen absolute Häufigkeit (wie oft es im Korpus vorkommt) und dessen relative Häufigkeit (wie oft es pro Million Wörter vorkommt).

Unterscheidet man, für eine erste Analyse, zwischen den beiden untersuchten Besteuerungsarten, nach denen die Texte ausgewählt wurden, steht die Erbschaftsbesteuerung stärker im Fokus der Berichterstattung, da das Stichwort „Erbschaftsteuer“ häufiger als „Vermögensteuer“ vorkommt. Bei den Bigrammen zeigt sich allerdings, dass Vermögensteuer mit „Wiedereinführung“ stark gekoppelt wird, etwas stärker als Erbschaftsteuer mit „Reform“. 
Tab. 2 Die vierzig häufigsten Wörter und Wortkombination in Überschriften, Leitsätzen und Volltexten aller Zeitungsartikel, Wort- und Bigramm-Häufigkeitslisten 2000 bis 2018

\begin{tabular}{|c|c|c|c|c|c|}
\hline Stichwort & $\begin{array}{l}\text { Absolute } \\
\text { Häufigkeit }\end{array}$ & $\begin{array}{l}\text { Relative } \\
\text { Häufigkeit }\end{array}$ & Bigramm & $\begin{array}{l}\text { Absolute } \\
\text { Häufigkeit }\end{array}$ & $\begin{array}{l}\text { Relative } \\
\text { Häufigkeit }\end{array}$ \\
\hline Jahr & 24.059 & 0,68 & Milliarde Euro & 5442 & 0,15 \\
\hline SPD & 23.945 & 0,68 & Million Euro & 2923 & 0,08 \\
\hline sagen & 21.195 & 0,60 & groß Koalition & 2725 & 0,08 \\
\hline Prozent & 19.789 & 0,56 & Angela Merkel & 1860 & 0,05 \\
\hline Euro & 19.694 & 0,56 & vergangen Jahr & 1840 & 0,05 \\
\hline mehr & 18.754 & 0,53 & Rot grün & 1400 & 0,04 \\
\hline geben & 17.337 & 0,49 & Gerhard Schröder & 1372 & 0,04 \\
\hline groß & 15.070 & 0,43 & Peer Steinbrück & 1120 & 0,03 \\
\hline neu & 13.865 & 0,39 & Sigmar Gabriel & 1090 & 0,03 \\
\hline gut & 13.693 & 0,39 & Euro Jahr & 949 & 0,03 \\
\hline gehen & 12.297 & 0,35 & SPD Grüne & 890 & 0,03 \\
\hline schon & 11.281 & 0,32 & $\begin{array}{l}\text { Nordrhein Westfa- } \\
\text { len }\end{array}$ & 858 & 0,02 \\
\hline Land & 11.183 & 0,32 & Bund Land & 845 & 0,02 \\
\hline Deutschland & 10.940 & 0,31 & nah Jahr & 830 & 0,02 \\
\hline kommen & 10.936 & 0,31 & sozial Gerechtigkeit & 830 & 0,02 \\
\hline hoch & 10.501 & 0,30 & kommend Jahr & 790 & 0,02 \\
\hline erst & 10.152 & 0,29 & Franz Müntefering & 764 & 0,02 \\
\hline Partei & 9731 & 0,27 & $\begin{array}{l}\text { Wiedereinführung } \\
\text { Vermögensteuer }\end{array}$ & 756 & 0,02 \\
\hline deutsch & 9657 & 0,27 & letzt Jahr & 718 & 0,02 \\
\hline immer & 9350 & 0,26 & steuern zahlen & 695 & 0,02 \\
\hline lassen & 8183 & 0,23 & $\begin{array}{l}\text { Reform Erbschaft- } \\
\text { steuer }\end{array}$ & 694 & 0,02 \\
\hline stehen & 7950 & 0,22 & immer mehr & 694 & 0,02 \\
\hline Erbschaftsteuer & 7726 & 0,22 & hoch steuern & 683 & 0,02 \\
\hline Grüne & 7426 & 0,21 & Union SPD & 683 & 0,02 \\
\hline Staat & 7378 & 0,21 & rund Milliarde & 676 & 0,02 \\
\hline Geld & 7311 & 0,21 & SPD Chef & 671 & 0,02 \\
\hline Milliarde & 7302 & 0,21 & vergangen Woche & 661 & 0,02 \\
\hline sehen & 7191 & 0,20 & schwarz gelb & 648 & 0,02 \\
\hline Unternehmen & 7072 & 0,20 & Jahr alt & 628 & 0,02 \\
\hline Zeit & 6959 & 0,20 & Horst Seehofer & 615 & 0,02 \\
\hline CDU & 6792 & 0,19 & Baden Württemberg & 612 & 0,02 \\
\hline Union & 6720 & 0,19 & Jahr lang & 600 & 0,02 \\
\hline Politik & 6560 & 0,19 & CDU CSU & 577 & 0,02 \\
\hline bleiben & 6548 & 0,18 & Hans Eichel & 568 & 0,02 \\
\hline Koalition & 6522 & 0,18 & mehr Geld & 552 & 0,02 \\
\hline ganz & 6512 & 0,18 & Göring Eckardt & 552 & 0,02 \\
\hline Mensch & 6382 & 0,18 & $\begin{array}{l}\text { Spitzensteuersatz } \\
\text { Prozent }\end{array}$ & 544 & 0,02 \\
\hline weit & 6355 & 0,18 & Oskar Lafontaine & 530 & 0,01 \\
\hline
\end{tabular}


Tab. 2 (Fortsetzung)

\begin{tabular}{llllll}
\hline Stichwort & $\begin{array}{l}\text { Absolute } \\
\text { Häufigkeit }\end{array}$ & $\begin{array}{l}\text { Relative } \\
\text { Häufigkeit }\end{array}$ & Bigramm & $\begin{array}{l}\text { Absolute } \\
\text { Häufigkeit }\end{array}$ & $\begin{array}{l}\text { Relative } \\
\text { Häufigkeit }\end{array}$ \\
\hline Regierung & 6140 & 0,18 & rot grün & 525 & 0,01 \\
steuern & 6015 & 0,17 & Wolfgang Schäuble & 507 & 0,01 \\
halten & 5880 & 0,17 & groß Vermögen & 499 & 0,01 \\
grün & 5810 & 0,17 & Schleswig Holstein & 483 & 0,01 \\
CSU & 5698 & 0,16 & deutsch Wirtschaft & 467 & 0,01 \\
sozial & 5679 & 0,16 & IG Metall & 460 & 0,01 \\
heute & 5614 & 0,16 & rund Prozent & 459 & 0,01 \\
Million & 5596 & 0,16 & SPD Linke & 457 & 0,01 \\
politisch & 5495 & 0,16 & öffentlich Dienst & 454 & 0,01 \\
liegen & 5452 & 0,16 & Hartz IV & 453 & 0,01 \\
Vermögensteuer & 5452 & 0,16 & SPD Vorsitzende & 449 & 0,01 \\
stark & 5418 & 0,15 & groß Teil & 445 & 0,01 \\
\hline
\end{tabular}

Tab. 2 legt außerdem dar, dass politikbezogene Worte häufig in der Berichterstattung vorkommen. So werden die beiden Besteuerungsarten eindeutig mit der SPD assoziiert (Platz 2 der Stichworte), von Politiker*innennamen angeführt (z. B. „Angela Merkel“ vor einer Zahl an SPD-Politiker*innen - von „Gerhard Schröder“ bis „Franz Müntefering“), sowie von politischen Parteien und Regierungskoalitionen dominiert (neben „SPD“ noch ,große Koalition“, „CSU“, „Union“ und „Grüne“, bis hin zu „schwarz gelb“). Die in den Häufigkeitslisten vorkommenden Schlüsselwörter „Unternehmen“, ,,deutsche Wirtschaft“, ,,soziale Gerechtigkeit“" und „Hartz IV“ zeigen den Zusammenhang zu gesellschaftspolitischen und wirtschaftlichen Debatten auf.

Weiters auffallend in obiger Häufigkeitsliste ist eine zeitliche und eine monetäre Dimension: Das Stichwort „Jahr“ wird am meisten gebraucht, weitere zeitbezogene Wortkombinationen finden sich unter den ersten 40 Bigrammen. Wie diese zeitlichen Worte gebraucht werden, ist damit noch nicht klar: Es kann sein, um die Termine einer Veränderung von Steuerreformen anzuzeigen, ebenso aber kann die konkrete Ausgestaltung der Steuern - zu zahlen binnen eines Jahres - angesprochen werden, um nur zwei von vielerlei Varianten zu nennen. Eins dieser Bigramme - „Euro Jahr“ - verknüpft die zeitliche mit der monetären Komponente: Letztere Komponente ist sowohl bei den Stichworten auf prominenten frühen Plätzen zu finden „Euro“ „Prozent“, noch klarer aber bei den Bigrammen auf Platz 1 und 2 „Milliarde Euro“ bzw. „Million Euro“.

Die häufigen Verben (Tab. 2) geben eher Dynamik an (,geben“, ,gehen“, „kommen“), bei den Adjektiven liegt die Vorstellung der gesteigerten Quantität zugrunde (,mehr“, „groß“, „,neu“, ,hoch“, ,immer“). In den Bigrammen verstärkt sich dies auf ,immer mehr“ und „Steuern zahlen“. Dass „Deutschland“ ein prominentes Wort darstellt, mag nicht weiter verwundern; dass besonders „Nordrhein Westphalen“, „Baden-Württemberg“ und „Schleswig Holstein“ häufig vorkommen, verweist auf die tonangebende Rolle von Akteuren aus diesen Bundesländern. 


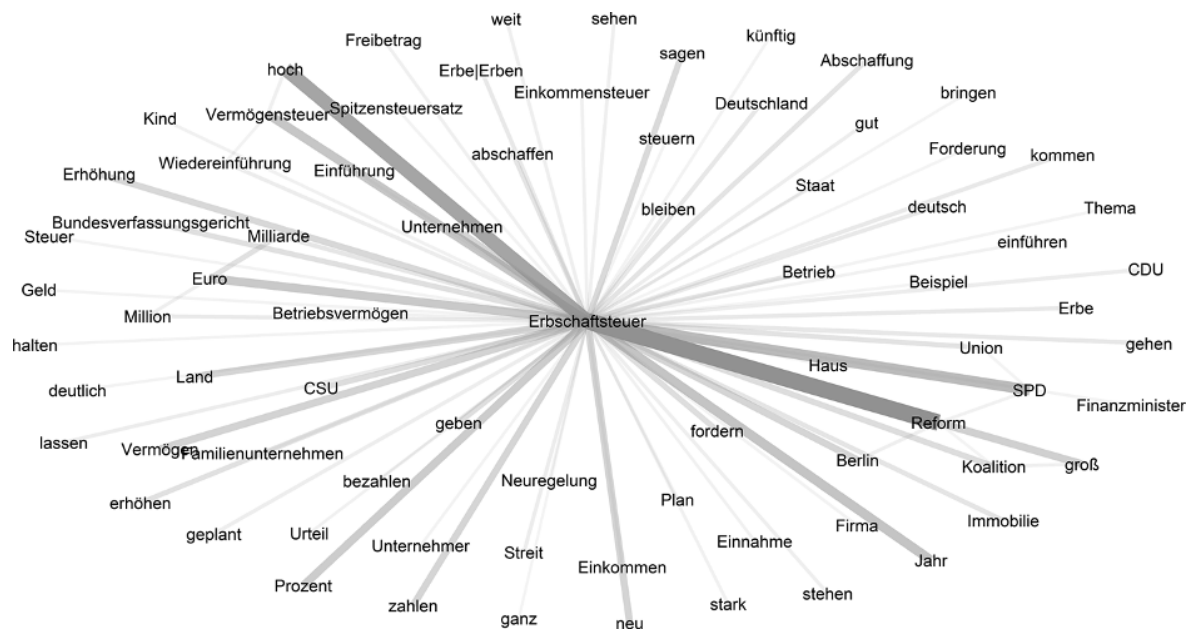

Abb. 285 häufigste Nomen, Verben, Pronomen und Adjektive, die im gleichen Satz mit ,Erbschaftssteuer“ vorkommen. Die Dicke der Verbindungslinien zeigt an, wie häufig die jeweiligen Wörter im gleichen Satz mit „Erbschaftssteuer“ vorkommen

Im nächsten Schritt werden wiederkehrende Substantive, Verben und Adjektive in unmittelbarer Nähe einiger ausgewählter Begriffe näher analysiert. In Abb. 2 sind die Nomen, Verben, Pronomen und Adjektive abgebildet, die regelmäßig im gleichen Satz mit dem Stichwort „Erbschaftssteuer“ vorkommen. Es lassen sich unterschiedliche Wortgruppen identifizieren. Die Wörter „Urteil“ und „Bundesverfassungsgericht“"weisen auf den Zusammenhang zu den beiden Verfassungsgerichtsurteilen von 2008 und 2014 hin; die anschließenden Reformprozesse und politischen Debatten beziehen sich auf Elemente bzw. Statements zu „Neuregelung“, „Streit“, „Reform“, Erhöhung, „Abschaffung“. Eine zweite Wortgruppe setzt sich aus den unterschiedlichen Vermögensarten zusammen, die (potentiell) unter die Erbschaftsbesteuerung fallen: „Firma“, „Betrieb(-svermögen)“, „Haus“, „Land“, „Immobilie“, „(Familien-)unternehmen“. Eine weitere Wortgruppe weist auf die konkrete Ausgestaltung der Steuer hin: „Freibetrag“, „Spitzensteuersatz“, „Einkommenssteuer“.

Hinsichtlich der Vermögenssteuer lassen sich bei regelmäßig wiederkehrenden Nomen, Verben, Pronomen und Adjektiven folgende Wortgruppen erkennen (Abb. 3). Die Forderung der Einführung bzw. Abschaffung der Vermögenssteuer ist wichtiger diskursiver Bestandteil der Berichterstattung (,(Wieder-)Einführung“, „Anhebung“, „Abschaffung“). Analog zur Erbschaftssteuer scheint auch bei der Vermögenssteuer der politische Raum zentraler Bestandteil der Berichterstattung zu sein - einerseits hinsichtlich der Debatte und Auseinandersetzung (,Diskussion“, „Forderung“, „Debatte“), andererseits auch bezogen auf politische Akteure (,Schröder“, „Sigmar Gabriel“, ,SPD“, „Grüne“, „Union“). Beide Netzwerkgrafiken zeigen auch, dass die Debatte um die Erbschaftssteuer und die der Vermögenssteuer eindeutig verknüpft ist; beide kommen regelmäßig in einem Satz vor.

Eine genauere Betrachtung der regelmäßig vorkommenden Wörter im gleichen Satz mit dem Stichwort Unternehmen zeigt, dass kleine, mittlere, bzw. mittelständische Unternehmen und Familienunternehmen im Vordergrund der Berichterstattung 


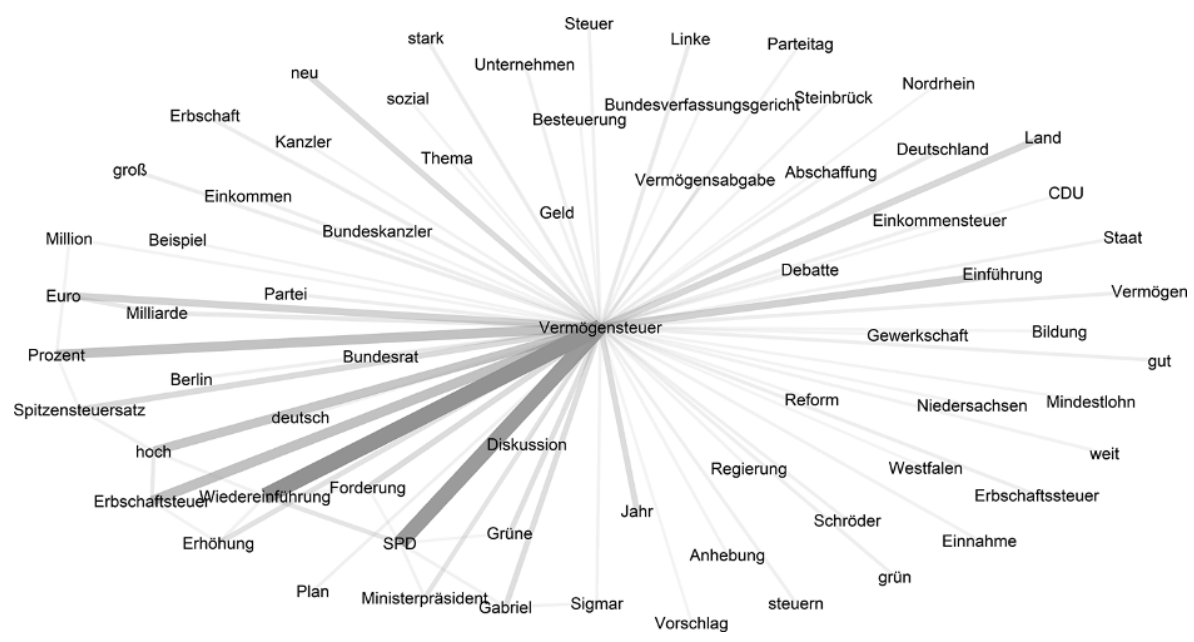

Abb. 385 häufigste Nomen, Verben, Pronomen und Adjektive, die im gleichen Satz mit „Vermögenssteuer“ vorkommen. Die Dicke der Verbindungslinien zeigt an, wie häufig die jeweiligen Wörter im gleichen Satz mit „Vermögenssteuer“ vorkommen

stehen (für die folgenden Analysen wird auf die grafische Darstellung im Text aus Platzgründen verzichtet). Zwar findet sich auch das Stichwort „groß“ regelmäßig in einem Satz mit Unternehmen; eine genauere Betrachtung zeigt allerdings, dass dies neben der Größenbeschreibung von Unternehmen vor allem dazu dient, die hohe Belastung von Unternehmen durch Erbschafts- und Vermögenssteuern darzustellen. Dieser häufige Verweis auf mittelständische Unternehmen reproduziert ein idealtypisches Bild von Firmen, impliziert ein inhaber*innengeführtes, traditionsbewusstes Wirtschaften (Lehrer und Schmid 2015). ${ }^{9}$ Weiterhin sind Beschreibung von Unternehmen wie Kapitalgesellschaft oder Großkonzern im Gegensatz zu den Familienunternehmen wesentlich seltener in der Berichterstattung vorzufinden. Darüber hinaus sind die Begriffe „Arbeitsplätze“, „Beschäftigte“ und „Mitarbeiter“ regelmäßige Wörter in unmittelbarer Nähe des Stichworts „Unternehmen“, was auf die Wichtigkeit der Debatte um mögliche Arbeitsplatzverluste durch Vermögens- und Erbschaftssteuern deutet.

Demgegenüber findet die diskursive Verknüpfung mit Gerechtigkeits- und Ungleichheitsfragen zwar durchaus statt - allerdings nur in einem geringeren und eng begrenzten Umfang. Die Analyse der wiederkehrenden Wörter in der Nähe der Stichworte „Gerechtigkeit“ und „Ungleichheit“ bringt vergleichsweise selten Begriffe wie „sorgen“, „Solidarität“, „Chancengleichheit“ oder „Gleichheit“ zu Tage.

In Übereinstimmung mit früheren Forschungsarbeiten (Grisold und Theine 2020) wird in den untersuchten Diskursen dem Staat und auch politischen Parteien und Politiker*innen eine ambivalente Rolle zugeschrieben. Einerseits wird die grundlegende Funktion des Staates, steuerfinanzierte öffentliche Güter bereitzustellen, an-

\footnotetext{
9 Das idealtypische Bild von Unternehmen als klein und mittelständisch, bzw. als familiengeführt zu charakterisieren ist ein zentraler Bestandteil der Kampagnen von Lobbyorganisationen gegen die Erbschaftsund Vermögensbesteuerung (Theine 2020).
} 
erkannt. Die KWIC-Analyse zeigt, dass häufige Wörter rund um das Schlüsselwort „Staat“ die Wörter „Einnahmen“, ,finanzieren“, „Gesellschaft“, ,,sozial“, ,,starken“ und ,handlungsfähigen“ sind, was auf die aktive und unterstützende Rolle des Staates hinzuweisen scheint. Gleichzeitig lassen die Wörter „,schlanken“, ,wenig(er)“, „sparen“ und „kosten“ auf eine eher ablehnende Haltung gegenüber der Rolle des Staates schließen. Hinsichtlich der politischen Parteien und Politiker*innen sind vergleichbare Muster zu erkennen. Zum einen lassen einige wiederkehrende Verben auf die aktive und gestaltende Rolle von Politiker*innen und Parteien schließen (,,sagen“, ,machen“, „lassen“), gleichzeitig weisen auch eine Reihe von Stichworten auf ablehnende Formulierungen hin (,warnen“, „dagegen“, „kritisieren“, „kaum“).

\subsection{Thematische Differenzen zwischen den analysierten Zeitungen}

Um die inhaltlichen Unterschiede zwischen den einzelnen Zeitungen zu analysieren, werden zunächst die häufigsten Nomina und Eigennamen nach Zeitungen näher betrachtet, um im Anschluss auf solche Wörter einzugehen, die nur in einzelnen Zeitungen besonders relevant sind.

Abb. 4 zeigt, dass - analog zu Tab. 2 - einige Stichworte über alle Zeitungen hinweg relevant sind. So ist beispielsweise das Stichwort „SPD“ bei allen untersuchten Zeitungen an erster Stelle; damit wird weiterhin unterstützt, dass diese Steuer-Reformthemen der SPD zugeschrieben werden. Ebenso sind die Begriffe „Land“, „Deutschland“ und „Partei“ in allen sieben Zeitungen recht weit oben auf den Ranglisten zu finden. Damit wird eine regionale bzw. nationale Zuständigkeit angesprochen und die Rolle der Politik über Parteien verfestigt. Bei einigen weiteren Stichworten zeigen sich aber auch leichte Unterschiede zwischen den Zeitungen: Während die Grünen in der $F A Z$ in der Häufigkeitsliste obenauf zu finden sind, ist die CDU bei Die Welt und die CSU in der Süddeutschen Zeitung prominent vertreten. Ähnliche Rangfolgen zeigen sich einerseits bei Die Zeit, FAZ und Süddeutscher Zeitung, andererseits auch bei Die Welt und Welt am Sonntag.

Interessant ist auch die nominelle Wertigkeit der beiden untersuchten Besteuerungsarten: Während die „Erbschaftsteuer“ eine unterschiedlich positionierte, aber doch hohe Häufigkeit vorweisen kann, ist die „Vermögensteuer“" nur in der FAZ viel diskutiert, in Die Welt und taz noch knapp unter den ersten $20 \mathrm{zu}$ finden. Die Unternehmens-Komponente ist nur der taz und dem Spiegel keinen jeweils prominenten Rang wert. Die monetäre Komponente generell ist in allen Zeitungen extrem prominent (,Geld“, „Milliarde“, „Million“). Auffallend ist weiterhin, dass Verteilungskomponenten dieser beiden Besteuerungsarten weder in Tab. 2 noch hier in Abb. 4 unter den häufigsten Worten genannt werden.

Um zusätzlich die Relevanz spezifischer Worte herauszuarbeiten, gibt Tab. 3 diejenigen Stichworte an, welche nur in einzelnen Zeitungen regelmäßig vorkommen, in anderen jedoch nicht. Ein Blick auf die Tabelle zeigt, dass unterschiedliche Worte in den sieben Zeitungen relevant sind. So greift zum Beispiel Die Zeit Entwicklungen und Themen im Zusammenhang mit Österreich auf, wie die Stichworte „ÖVP“ und „SPÖ“ zeigen. In Die Welt, Welt am Sonntag und FAZ sind einige unternehmensbezogene Wörter vorhanden, die den besonders ausgeprägten ökonomischen Fokus in den drei Zeitungen zeigt. Die Welt rekurriert verstärkt auf die Rolle der Immobilien 


\begin{tabular}{|c|c|c|c|c|c|c|c|}
\hline Stichwort & $\begin{array}{c}\text { Süddeutsche } \\
\text { Zeitung }\end{array}$ & Der Spiegel & Die Welt & DIE ZEIT & FAZ & taz & $\begin{array}{l}\text { Welt am } \\
\text { Sonntag }\end{array}$ \\
\hline SPD & 1 & 1 & 1 & 1 & 1 & 1 & 1 \\
\hline Land & 2 & 3 & 2 & 3 & 2 & 7 & 3 \\
\hline Erbschaftsteuer & 3 & & 5 & 18 & 13 & 15 & 5 \\
\hline Deutschland & 4 & 4 & 3 & 2 & 4 & 4 & 2 \\
\hline CSU & 5 & & 12 & & & & 14 \\
\hline Partei & 6 & 2 & 6 & 8 & 7 & 3 & 4 \\
\hline Geld & 7 & 6 & 20 & 5 & 16 & 6 & 16 \\
\hline Unternehmen & 8 & & 10 & 10 & 6 & & 6 \\
\hline Milliarde & 9 & 14 & 13 & 13 & 9 & 5 & 18 \\
\hline Staat & 10 & 11 & 15 & 4 & 8 & 14 & 11 \\
\hline Union & 11 & 7 & 7 & & & 11 & 9 \\
\hline Koalition & 12 & 10 & 8 & & & 12 & 8 \\
\hline Reform & 13 & & 11 & & & & \\
\hline Regierung & 14 & 12 & 16 & 12 & 14 & 20 & \\
\hline Million & 15 & & & 15 & 17 & 19 & \\
\hline steuern & 16 & & & 9 & 11 & & 17 \\
\hline Mensch & 17 & 9 & & 6 & & 13 & 10 \\
\hline CDU & 18 & 16 & 4 & & 10 & 8 & 12 \\
\hline Kind & 19 & & 19 & & & & 15 \\
\hline Vermögen & 20 & & & 11 & 15 & & \\
\hline Merkel & & 5 & 17 & & & & 19 \\
\hline Politik & & 8 & 14 & 7 & 12 & 10 & 7 \\
\hline Grüne & & 13 & 9 & & 5 & 2 & 13 \\
\hline Gabriel & & 15 & & & & & \\
\hline Steinbrück & & 17 & & & & & \\
\hline Frage & & 18 & & 14 & & & \\
\hline Tag & & 19 & & & & & \\
\hline Kanzler & & 20 & & & & & \\
\hline Einkommen & & & & 20 & & & \\
\hline FDP & & & & & 19 & 16 & 20 \\
\hline grün & & & & & & 17 & \\
\hline Leute & & & & 19 & & & \\
\hline Linke & & & & & & 9 & \\
\hline Mann & & & & 17 & & & \\
\hline Vermögensteuer & & & 18 & & 3 & 18 & \\
\hline Vorsitzende & & & & & 20 & & \\
\hline Wirtschaft & & & & 16 & 18 & & \\
\hline
\end{tabular}

Abb. 4 Rangfolge der häufigsten 20 Nomen und Eigennamen in den sieben Zeitungen

(ein gern gebrauchtes Argument, die extreme Vermögensschere in Deutschland zu relativieren bzw. zu rechtfertigen), in der FAZ ist eine Reihe abwertender Worte auffallend (,Zwangsanleihe“, „Unrechtsstaat“, ,Reuegebühr“), wohingegen in die Welt am Sonntag spezifische Worte genutzt wurden, die mit der Rolle von Unternehmen in den Besteuerungsarten im Zusammenhang stehen (,Unternehmenssteuerreform, Unternehmenssteuer").

In der taz sind einige Wörter einzigartig, die den Zusammenhang zwischen Erbschafts- und Vermögenssteuern mit Krisenentwicklungen (,Eurokrise“, „Globalisierungskritiker“) sowie mit dem Klimawandel (,CO2“) aufzeigen, hier findet sich auch 
Tab. 3 Spezifische Worte in der Berichterstattung einzelner Zeitungen

\begin{tabular}{|c|c|}
\hline Zeitung & Wörter \\
\hline Der Spiegel & $\begin{array}{l}\text { Habeck, Böhmermann, Ecclestone, Roßmann, Würth, Netzwerker, DPA, Grass, Sinclair, } \\
\text { Redakteur, Occupy, Baerbock, Biografie, achtzig, Montebourg, Bultmann, Mack, Op- } \\
\text { penheim, Strategen, Tagesschau, Sarkozy, Elysée, nicken, Spitzengenosse, Unterschicht, } \\
\text { vergangen }\end{array}$ \\
\hline Die Welt & $\begin{array}{l}\text { Hauptversammlung, DIHK, Abgeltungssteuer, Immobilienwirtschaft, Monaco, IW, Mak- } \\
\text { ler, Söder, Habeck, Steuerzahlerbund, Unternehmensnachfolge, Selbstständige, notariell, } \\
\text { Veräußerungsgewinne, Immobilienerbe, Wirtschaftsflügel, Sotheby’s, vermietet, Finanz- } \\
\text { marktdaten, Betriebserbe, Steuerzahlergedenktag }\end{array}$ \\
\hline Die Zeit & $\begin{array}{l}\text { SPÖ, ÖVP, Gauweiler, achtzig, Voves, Selbstständige, Zamperoni, Paqué, Rawls, Samm- } \\
\text { lungsbewegung, Unternehmenssteuer, Schellhorn, Sotheby’s, Asmussen, Unterschicht }\end{array}$ \\
\hline FAZ & $\begin{array}{l}\text { Abgeltungssteuer, Ooyen, Selbständige, demographisch, DIHK, Palmer, Georgi, Amnes- } \\
\text { tieerklärung, Veräußerungsgewinne, achtzig, Einbeziehung, potentiell, Substanzbesteue- } \\
\text { rung, Wazir, GEW, Mitgliederbegehren, Zwangsanleihe, Atkinson, Familiengesellschaft, } \\
\text { Unrechtsstaat, Reuegebühr }\end{array}$ \\
\hline $\begin{array}{l}\text { Süddeutsche } \\
\text { Zeitung }\end{array}$ & $\begin{array}{l}\text { Söder, Oberbayern, Abgeltungssteuer, Einkommenssteuer, Friedrichs, Selbständige, } \\
\text { Starnberg, Aiwanger, Podemos, DIHK, Unternehmenssteuer, Schenkungssteuer, Bern- } \\
\text { hardt, Bodenrichtwert, Verschonungsregel, Vermögensübertragung, Steuerfreibetrag, } \\
\text { Erbvertrag, Vermögensmanager }\end{array}$ \\
\hline taz & $\begin{array}{l}\text { Habeck, Hofreiter, Umverteilung, Troost, WählerInnen, Giegold, GAL, Paritätisch, Eu- } \\
\text { rokrise, Linksfraktion, CO2, Globalisierungskritiker, Butterwegge, Friedrichshain, Fe- } \\
\text { minismus, GEW, rechtspopulistisch, aufkommensneutral, Volksbegehren, Innensenator, } \\
\text { Arbeitnehmerkammer, MieterInnen, GenossInnen }\end{array}$ \\
\hline $\begin{array}{l}\text { Welt am } \\
\text { Sonntag }\end{array}$ & $\begin{array}{l}\text { Bisky, Beuys, Hennerkes, Fachanwalt, Ferienimmobilie, IW, Staatsregierung, Unterneh- } \\
\text { menssteuerreform, Grundbuch, Unternehmenssteuer, Zweitmarkt, } \\
\text { vermietet, Arbeitslosenzahl, notariell, Stiftungszweck, Altersvorsorgevertrag, Spitzen- } \\
\text { steuersatzzahler }\end{array}$ \\
\hline
\end{tabular}

das einzige Mal das Wort „Umverteilung“. Die $S Z$ scheint verstärkt auf Details der Ausgestaltung der Erbschafts- und Vermögenssteuer einzugehen, wie einige Stichworte nahelegen (,Bodenrichtwert“, „Vermögensübertragung“", „Steuerfreibetrag“).

An die Ergebnisse aus dem vorherigen Abschnitt anschließend, sind auch hier einige Namen von Politiker*innen vorhanden; allerding solche, die die im gesamten Korpus weniger relevant sind („Habeck“, „Söder“, „,Voves“, „Wazir“, „Sarkozy“). Aus Tab. 3 wird auch deutlich, dass Arbeitnehmer*innenvertretungen selten in der Berichterstattung vorkommen, wenn, dann hauptsächlich in Form der GEW (Gewerkschaft Erziehung und Wissenschaft), dies in der Berichterstattung der FAZ und der taz.

\subsection{Anlässe verstärkter Berichterstattung}

Wenn auch generell die Berichterstattung zu den Themen Erbschafts- und Vermögenssteuer nicht besonders ausgeprägt ist, so lassen sich doch vier Zeitperioden identifizieren, in denen intensiver darüber berichtet wurde. Diese vier Perioden werden im Folgenden näher thematisiert und dies im Kontext der Anlässe der Berichterstattung, so dass deutlich wird, warum und in welchem Zusammenhang die Erbschaftsund Vermögenssteuern in den Medien intensiver als sonst thematisiert wurden. Neben einer grafischen Darstellung der Anzahl der Artikel in den vier Perioden werden 

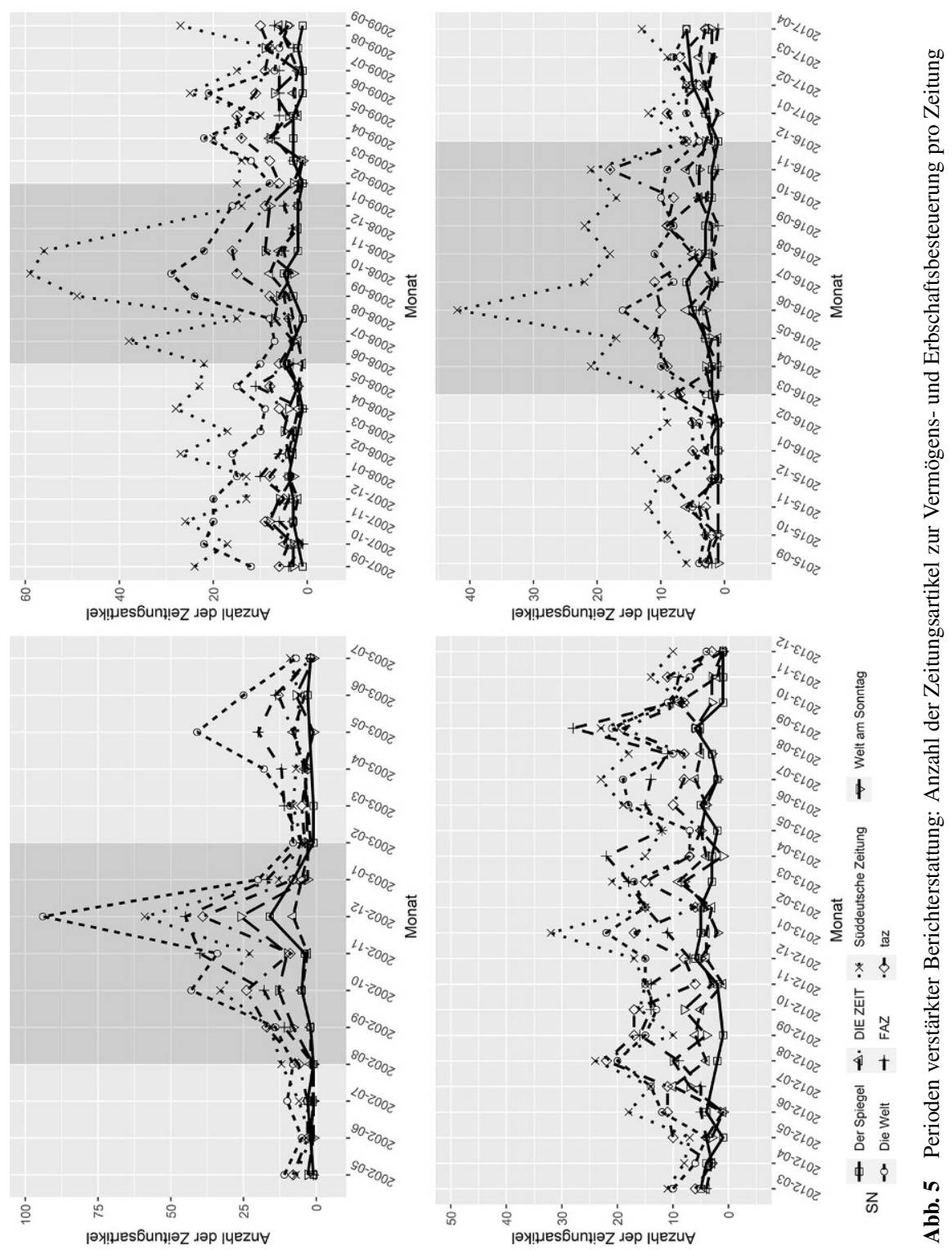
dazu die Inhalte mittels einer Analyse der häufigsten Wörter und Wortkombinationen untersucht (Anhang, Tab. 4, 5, 6 und 7).

Eine genauere Betrachtung des ersten Zeitraums zeigt, dass insbesondere die Monate August 2002 bis Januar 2003 (siehe grau markierter Bereich in Abb. 5) eine intensive Berichterstattung aufweisen. Die Worthäufigkeitslisten der zwischen August 2002 und Januar 2003 veröffentlichten Artikel (Anhang, Tab. 4) zeigen, dass die Vermögensbesteuerung in diesem Zeitraum im Mittelpunkt stand (,Vermögensteuer" ist das häufigste Schlagwort in diesen Monaten). Darüber hinaus sind mehrere politikbereichsbezogene Wörter wiederkehrend vorhanden (wie zum Beispiel ,,rotgrün“, „SPD“, „Regierung“ und „,nach der Bundestagswahl“). Außerdem werden führende Politiker einzelner Bundesländer (,Niedersachsens Ministerpräsident Gabriel“, ,Ministerpräsident Roland Koch“, „Edmund Stoiber“, ,Wolfgang Clement“) und Politiker auf Bundesebene (,Kanzler Schröder“, „Fraktionschef Müntefering“) genannt. Eine Reihe häufiger Wortkombinationen verweist auf heftige Kontroversen in der politischen Arena: „Streit um die Vermögenssteuer“, „Kanzler umkurvt Vermögensteuer“, „Schröder spricht Machtwort“, ,Ver.di droht““. Zusammenfassend zeigen die Häufigkeitslisten damit, dass die Berichterstattung in diesem Zeitraum auf die Debatte und Kontroversen um Vermögensbesteuerung ausgerichtet ist und dabei nicht nur, aber besonders auch innerhalb der (damaligen Regierungspartei) SPD Anlass war.

Im zweiten Zeitraum ist speziell die Berichterstattung in der $S Z$ (mit 40 bis $60 \mathrm{Ar}-$ tikeln pro Monat) zwischen Mai 2008 und Januar 2009 besonders intensiv (siehe grau markierter Bereich in Abb. 5). Die Worthäufigkeitslisten (Anhang, Tab. 5) zeigen eindeutig, dass sich die Berichterstattung in diesem Zeitraum auf die Erbschaftssteuer konzentriert. Auch hier stehen politikfeldbezogene Wörter und Wortkombinationen (,SPD“, „Union“, „große Koalition“, „Horst Seehofer“, „Bundeskanzlerin Angel Merkel“") im Vordergrund. In diesem Zeitraum ist es insbesondere die Debatte in und zwischen den regierenden Parteien der großen Koalition darüber, wie die durch das Urteil des Bundesverfassungsgerichts notwendig gewordene Erbschaftssteuerreform ausgestaltet sein soll, die im Vordergrund der Berichterstattung steht.

Im Gegensatz zu den drei anderen Perioden ist der dritte Zeitraum (März 2012 bis Dezember 2013) durch eine relativ lange, intensive Berichterstattung ohne eindeutigen Höhepunkt gekennzeichnet. Hierbei werden sowohl die Erbschafts- als auch die Vermögensbesteuerung diskutiert (siehe Anhang, Tab. 6). Neben den Sozialdemokraten (SPD) und Christdemokraten (Union) gehören in dieser Zeitperiode die Grünen (Bündnis 90/Die Grünen) zu den am häufigsten genannten Parteien. Wir nehmen an, dass letzteres aufgrund der grünen Wahlkampfforderung im Rahmen der Bundestagswahl 2013, die Vermögenssteuer wiedereinzuführen, zustande kommt. Die Schlagwörter „Hollande“ und „Depardieu“ implizieren zudem, dass die Frage der Vermögensbesteuerung häufig mit dem politischen Reformprozess in Frankreich verbunden wird: François Hollande führte 2012, als französischer Präsident, die Vermögensbesteuerung wieder ein. Dies wurde äußerst kontrovers diskutiert, und führte - unter anderem - dazu, dass der Schauspieler Gérard Depardieu medienwirksam androhte, in diesem Falle die französische Staatsbürgerschaft abzugeben (Clift und McDaniel 2017; Süddeutsche Zeitung 2013). 
Während der vierten Periode gibt es zwischen Februar und November 2016 eine Hochphase in der Berichterstattung (siehe Grauzone in Abb. 5). „Erbschaftsteuer“, „Reform der Erbschaftsteuer“ und „Neuregelung der Erbschaftsteuer“ sind hier die am häufigsten verwendeten Wörter und Wortkombinationen (Anhang, Tab. 7). Darüber hinaus sind die Schlagworte ,streiten“, ,im Streit um“ und „Kompromiss“ oft vorzufinden. Der Fokus liegt damit klar auf der Erbschaftsbesteuerung und darin auf den Auseinandersetzungen und Kompromissbemühungen der Regierungskoalition SPD und CDU/CSU anlässlich der Erbschaftssteuerreform, die durch das zweite Urteil des Bundesverfassungsgerichts notwendig wurde.

\section{Fazit}

Welches Bild wird nun von der Vermögens- und Erbschaftsbesteuerung in der massenmedialen öffentlichen Diskussion im untersuchten Zeitraum gezeichnet und befördert?

Im Beitrag wurden diskursanalytisch die Rolle und die Standpunkte von Massenmedien $\mathrm{zu}$ neuen (oder oftmals auch: in früheren Zeiten bereits in Kraft befindlichen) Formen der Verteilungspolitik untersucht. Exemplarisch herausgegriffen wurden sieben deutsche Qualitätszeitungen, um die Berichterstattung zur Vermögens- und Erbschaftsbesteuerung in Deutschland in den ersten beiden Jahrzehnten des 21. Jahrhunderts zu analysieren. Dazu wurden knapp 10.000 Zeitungsartikel hinsichtlich ihrer textlichen Ausrichtung untersucht, wobei eine quantitative Analyse vorgenommen wurde, die über Anzahl an Artikeln, häufig vorkommenden Worten bzw. Wortkombinationen erfolgte.

Die Ergebnisse dieses Beitrags können in vier zentralen Punkten zusammengefasst werden: Erstens zeigt die Anzahl der Zeitungsartikel im Zeitverlauf, dass die Intensität der Berichterstattung auf niedrigem Niveau verläuft und im Zeitablauf durchaus schwankend erfolgt. Angesichts der Dringlichkeit des Themas, welche von verschiedensten und durchaus prominenten Sozialwissenschaftler*innen und ÖkonomInnen wie Krugman (2017) oder Stiglitz (2019) immer wieder vorgebracht wird, sowie angesichts der stark ausgeprägten Vermögensungleichheit in Deutschland ist dies ein äußerst bemerkenswerter Befund. Eine systematische Zunahme der Berichterstattung über den Untersuchungszeitraum ist nicht festzustellen - durchaus im Gegensatz zu der Berichterstattung zu verwandten Themen wie z.B. der ökonomischen Ungleichheit (Schröder und Vietze 2015).

Zweitens liegt der diskursive Fokus der Berichterstattung auf der (partei)politischen Ebene, weist eine eindeutig zeitbezogene sowie monetäre Dimension auf, und lässt einen, wenn auch eher schwachen, Zusammenhang mit gesellschaftspolitischen und wirtschaftlichen Debatten erkennen. Drittens verstärkt die Analyse einzelner, ausgewählter Zeiträume den Eindruck, dass der (partei)politische Diskurs im Vordergrund steht, da Kontroversen im politischen Raum und Debatten um die Reform der Erbschaftssteuer die dominanten Anlässe der Berichterstattung darstellen. Viertens lässt sich eine ähnlich gelagerte Ausrichtung der Berichterstattung in allen Zeitungen erkennen; einige Differenzen ergeben sich hinsichtlich unternehmensbezogener Aspekte (Die Welt, Welt am Sonntag und FAZ), Details zur Ausgestaltung 
der Steuern (Süddeutsche Zeitung) sowie dem Zusammenhang zwischen Erbschaftsund Vermögenssteuern mit Krisenentwicklungen und dem Klimawandel (taz). Diese Ergebnisse schließen an bisherige empirische Forschungen an, insbesondere an Analysen, die zeigen, dass sich die Berichterstattung oftmals an den Debatten und Kontroversen innerhalb des politischen Raums orientiert (u.a. Krüger 2019) bzw. Unterschiede in der Ausrichtung sich tendenziell entlang der politische Orientierung der Zeitungen ergeben (u.a. Lichtenstein et al. 2016).

Die gesellschaftspolitische Relevanz der vorliegenden Ergebnisse ergeben sich insbesondere, wenn der Einfluss der Medien auf die öffentliche Meinung sowie die individuellen Einstellungen zur ökonomischen Ungleichheit und zu Umverteilungsmaßnahmen berücksichtigt wird (siehe dazu u. a.: Epp und Jennings 2020; Grisold und Theine 2020). Aus den Ergebnissen der vorliegenden Studie lassen sich aufgrund der recht geringen Intensität, der Dominanz der (partei)politischen Ebene sowie der eher schwach ausgeprägten gesellschaftspolitischen Dimension der Berichterstattung tendenziell eher skeptische bzw. ablehnende Einstellungen gegenüber Erbschafts- und Vermögenssteuer schlussfolgern. Existierende Studien zu Umverteilungspräferenzen in Deutschland lassen diesen Schluss zu, wobei die Rolle der Medien hier bisher nicht systematisch erforscht worden ist (Barnes 2021; Beckert und Arndt 2017). Eine weitere Dimension der gesellschaftspolitischen Relevanz ergibt sich mit Bezug auf Fastenrath et al. (2021), die zeigen, dass Politiker*innen auf ablehnende und skeptische Mediendiskurse als ein wichtiges Hindernis für die höhere Besteuerung von Vermögen und Erbschaften hinweisen. Im Licht der hier vorgelegten Ergebnisse lassen sich Mediendiskurse zur Vermögens- und Erbschaftsbesteuerung als tendenzielle Barrieren gegen progressive Umverteilungspolitik verstehen.

Letztlich ergeben sich aus diesem Beitrag weiterer Forschungsbedarf zur Rolle der (Massen)medien in der Vermittlung von Vermögensungleichheit und Umverteilungsmaßnahmen. Komplementär zum vorliegenden Beitrag könnten die TV- und Radioberichterstattung sowie Debatten in sozialen Medien zu Erbschafts- und Vermögenssteuern für die Analyse herangezogen werden. Dies würde auch einen systematischen Vergleich unterschiedlicher Medientypen ermöglichen. Außerdem könnten qualitative Studien die Einschränkungen der vorliegenden Studie wettmachen, indem für ausgewählte Perioden (z. B. zu der Diskussion um die Vermögensabgabe zur Finanzierung der angestiegenen Staatsschulden im Rahmen der Coronapandemie) vertiefte Analysen der diskursiven Konstruktion von Vermögen und Erbschaften und ihrer Besteuerung durchgeführt werden. 


\section{Anhang}

Tab. 4 Wort- und Bigramm-Häufigkeitslisten der dreißig häufigsten Wörter und Wortkombination in den Überschriften und Leitsätzen der Zeitungsartikel (August 2002 bis Januar 2003)

\begin{tabular}{|c|c|c|c|c|}
\hline Häufige Wörter & $n$ & Häufige Bigramme & & $n$ \\
\hline vermögensteuer & 100 & sigmar & gabriel & 18 \\
\hline schröder & 82 & öffentlichen & dienst & 16 \\
\hline spd & 66 & gerhard & schröder & 11 \\
\hline gabriel & 48 & höhere & steuern & 9 \\
\hline kanzler & 46 & kanzler & schröder & 7 \\
\hline union & 32 & franz & müntefering & 6 \\
\hline steuern & 29 & frank & bsirske & 5 \\
\hline rotgrün & 24 & ministerpräsident & sigmar & 5 \\
\hline koch & 23 & niedersachsens & ministerpräsident & 5 \\
\hline steuererhöhungen & 23 & roland & koch & 5 \\
\hline deutschland & 22 & wolfgang & clement & 5 \\
\hline clement & 21 & 100 & milliarden & 4 \\
\hline geld & 21 & edmund & stoiber & 4 \\
\hline gewerkschaften & 21 & gerhard & bökel & 4 \\
\hline meinungsseite & 21 & gewerkschaft & verdi & 4 \\
\hline ministerpräsident & 19 & hessens & ministerpräsident & 4 \\
\hline öffentlichen & 19 & kasse & bitten & 4 \\
\hline dienst & 18 & verdi & droht & 4 \\
\hline höhere & 18 & dramatischer & einbruch & 3 \\
\hline pds & 18 & erbschaftsteuer & teil & 3 \\
\hline regierung & 18 & finanzminister & eichel & 3 \\
\hline sigmar & 18 & fraktionschef & müntefering & 3 \\
\hline zinssteuer & 18 & gabi & zimmer & 3 \\
\hline erbschaftsteuer & 17 & große & koalition & 3 \\
\hline müntefering & 17 & heftige & kritik & 3 \\
\hline verdi & 17 & heide & simonis & 3 \\
\hline milliarden & 16 & höheren & abgaben & 3 \\
\hline cdu & 15 & kanzler & umkurvt & 3 \\
\hline gerhard & 15 & lehnt & vermögensteuer & 3 \\
\hline koalition & 15 & ministerpräsident & roland & 3 \\
\hline
\end{tabular}


Tab. 5 Wort- und Bigramm-Häufigkeitslisten der dreißig häufigsten Wörter und Wortkombination in den Überschriften und Leitsätzen der Zeitungsartikel (Mai 2008 bis Januar 2009)

\begin{tabular}{|c|c|c|c|c|}
\hline Häufige Wörter & $n$ & Häufige Bigramme & & $n$ \\
\hline csu & 292 & horst & seehofer & 65 \\
\hline erbschaftsteuer & 283 & angela & merkel & 54 \\
\hline spd & 258 & großen & koalition & 48 \\
\hline union & 171 & große & koalition & 32 \\
\hline koalition & 153 & erwin & huber & 31 \\
\hline reform & 127 & peer & steinbrück & 26 \\
\hline seehofer & 115 & günther & beckstein & 23 \\
\hline merkel & 92 & kurt & beck & 20 \\
\hline cdu & 89 & frankwalter & steinmeier & 19 \\
\hline bayern & 88 & bundeskanzlerin & angela & 18 \\
\hline münchen & 84 & georg & fahrenschon & 18 \\
\hline fdp & 81 & oskar & lafontaine & 17 \\
\hline horst & 70 & steinbrück & spd & 15 \\
\hline erben & 68 & martin & zeil & 13 \\
\hline partei & 67 & ministerpräsident & günther & 13 \\
\hline großen & 64 & peter & ramsauer & 13 \\
\hline huber & 59 & peter & struck & 12 \\
\hline große & 57 & csuchef & horst & 11 \\
\hline angela & 56 & finanzminister & peer & 11 \\
\hline deutschen & 56 & franz & müntefering & 11 \\
\hline ministerpräsident & 56 & michael & glos & 11 \\
\hline deutschland & 53 & roland & koch & 10 \\
\hline erbschaftssteuer & 52 & volker & kauder & 10 \\
\hline steuern & 52 & bundesfinanzminister & peer & 9 \\
\hline kanzlerin & 51 & klaus & michael & 9 \\
\hline streit & 51 & merkel & $\mathrm{cdu}$ & 9 \\
\hline steuer & 50 & michael & groll & 9 \\
\hline bayerischen & 49 & ministerpräsident & horst & 9 \\
\hline dokument & 48 & axel & springer & 8 \\
\hline politik & 46 & csuchef & erwin & 8 \\
\hline
\end{tabular}


Tab. 6 Wort- und Bigramm-Häufigkeitslisten der dreißig häufigsten Wörter und Wortkombination in den Überschriften und Leitsätzen der Zeitungsartikel (März 2012 bis Dezember 2013)

\begin{tabular}{|c|c|c|c|c|}
\hline Häufige Wörter & $n$ & Häufige Bigramme & & $n$ \\
\hline spd & 196 & peer & steinbrück & 55 \\
\hline steuern & 131 & höhere & steuern & 33 \\
\hline grünen & 130 & angela & merkel & 30 \\
\hline deutschland & 118 & françois & hollande & 30 \\
\hline steinbrück & 103 & jürgen & trittin & 26 \\
\hline grüne & 94 & gérard & depardieu & 20 \\
\hline union & 94 & sigmar & gabriel & 18 \\
\hline deutschen & 91 & wolfgang & schäuble & 15 \\
\hline vermögensteuer & 82 & horst & seehofer & 14 \\
\hline geld & 76 & steuern & zahlen & 14 \\
\hline partei & 76 & martin & greive & 13 \\
\hline merkel & 74 & dorothea & siems & 12 \\
\hline zahlen & 69 & jochen & gaugele & 12 \\
\hline fdp & 64 & große & koalition & 11 \\
\hline frankreich & 62 & soziale & gerechtigkeit & 11 \\
\hline milliarden & 61 & stefan & reinecke & 10 \\
\hline reichensteuer & 59 & matthias & kamann & 9 \\
\hline peer & 58 & präsident & françois & 9 \\
\hline koalition & 57 & spdkanzlerkandidat & peer & 9 \\
\hline politik & 57 & ulf & poschardt & 9 \\
\hline höhere & 55 & ulrich & schulte & 9 \\
\hline $\operatorname{csu}$ & 54 & barack & obama & 8 \\
\hline hollande & 54 & claudia & kade & 8 \\
\hline $\mathrm{cdu}$ & 53 & daniel & friedrich & 8 \\
\hline regierung & 53 & deutschen & industrie & 8 \\
\hline große & 50 & deutschland & kompakt & 8 \\
\hline reiche & 50 & friedrich & sturm & 8 \\
\hline staat & 50 & katrin & göringeckardt & 8 \\
\hline erbschaftsteuer & 49 & reichtum & besteuern & 8 \\
\hline
\end{tabular}


Tab. 7 Wort-, Bigramm- und Trigramm-Häufigkeitslisten der dreißig häufigsten Wörter und Wortkombination in den Überschriften und Leitsätzen der Zeitungsartikel (Februar 2016 bis November 2016)

\begin{tabular}{|c|c|c|c|c|}
\hline Häufige Wörter & $n$ & Häufige Bigramme & & $n$ \\
\hline erbschaftsteuer & 165 & horst & seehofer & 36 \\
\hline grünen & 118 & angela & merkel & 31 \\
\hline spd & 113 & sigmar & gabriel & 29 \\
\hline koalition & 90 & große & koalition & 22 \\
\hline reform & 71 & winfried & kretschmann & 16 \\
\hline seehofer & 69 & großen & koalition & 15 \\
\hline $\operatorname{csu}$ & 63 & wolfgang & schäuble & 14 \\
\hline partei & 60 & daniel & friedrich & 13 \\
\hline grüne & 58 & friedrich & sturm & 13 \\
\hline merkel & 58 & donald & trump & 12 \\
\hline deutschland & 54 & martin & greive & 12 \\
\hline große & 48 & schäuble & $\mathrm{cdu}$ & 12 \\
\hline bundesrat & 45 & claudia & kade & 9 \\
\hline streit & 45 & seehofer & csu & 9 \\
\hline steuern & 43 & ulrich & schulte & 9 \\
\hline cdu & 42 & jürgen & trittin & 8 \\
\hline gabriel & 42 & andrea & nahles & 7 \\
\hline geld & 42 & anton & hofreiter & 7 \\
\hline bundestag & 41 & markus & söder & 7 \\
\hline erben & 41 & robert & habeck & 7 \\
\hline union & 39 & simone & peter & 7 \\
\hline gut & 38 & steuern & zahlen & 7 \\
\hline gesetz & 37 & arbeitsplätze & erhalten & 6 \\
\hline horst & 37 & bundesfinanzminister & wolfgang & 6 \\
\hline kompromiss & 37 & cem & özdemir & 6 \\
\hline angela & 32 & csuchef & horst & 6 \\
\hline vermögensteuer & 32 & geplante & reform & 6 \\
\hline milliarden & 31 & hannes & koch & 6 \\
\hline thema & 31 & merkel & $\mathrm{cdu}$ & 6 \\
\hline
\end{tabular}

Danksagung Die Autor*innen danken Daniel Grabner, Benjamin Ferschli, Nora Waitkus, Stefan Wallaschek sowie eine*r anonymen Gutachter*in für hilfreiche Diskussionen, Kommentare und konstruktive Hinweise, die geholfen haben unseren Beitrag zu verbessern. Dank auch an Victor Perez Delgado für seine Forschungsunterstützung. Dieser Beitrag wurde von der Otto-Brenner-Stiftung unterstützt [Projektnummer 2019-200].

Funding Open access funding provided by Vienna University of Economics and Business (WU).

Open Access Dieser Artikel wird unter der Creative Commons Namensnennung 4.0 International Lizenz veröffentlicht, welche die Nutzung, Vervielfältigung, Bearbeitung, Verbreitung und Wiedergabe in jeglichem Medium und Format erlaubt, sofern Sie den/die ursprünglichen Autor(en) und die Quelle ordnungsgemäß nennen, einen Link zur Creative Commons Lizenz beifügen und angeben, ob Änderungen vorgenommen wurden. 
Die in diesem Artikel enthaltenen Bilder und sonstiges Drittmaterial unterliegen ebenfalls der genannten Creative Commons Lizenz, sofern sich aus der Abbildungslegende nichts anderes ergibt. Sofern das betreffende Material nicht unter der genannten Creative Commons Lizenz steht und die betreffende Handlung nicht nach gesetzlichen Vorschriften erlaubt ist, ist für die oben aufgeführten Weiterverwendungen des Materials die Einwilligung des jeweiligen Rechteinhabers einzuholen.

Weitere Details zur Lizenz entnehmen Sie bitte der Lizenzinformation auf http://creativecommons.org/ licenses/by/4.0/deed.de.

\section{Literatur}

Adermon, Adrian, Mikael Lindahl, und Daniel Waldenström. 2018. Intergenerational wealth mobility and the role of inheritance: Evidence from multiple generations. The Economic Journal 128(612):F482-F513.

Augstein, Franziska. 2005. Einfalt oder Vielfalt? Von Pressekonzentration und Selbstgleichschaltung im Zeitungswesen. Blätter für deutsche und internationale Politik 50(12):1492-1502.

Bach, Stefan. 1997. Steuerreform in Deutschland. Vierteljahrshefte zur Wirtschaftsforschung 66(3/4): 291-316.

Bach, Stefan. 2016. Erbschaftsteuer, Vermögensteuer oder Kapitaleinkommensteuer: Wie sollen hohe Vermögen stärker besteuert werden? DIW Berlin Discussion Paper, Bd. 1619. Berlin: Deutsches Institut für Wirtschaftsforschung.

Bach, Stefan, und Martin Beznoska. 2012. Aufkommens- und Verteilungswirkungen einer Wiederbelebung der Vermögensteuer. DIW Berlin: Politikberatung kompakt, Bd. 68. Berlin: Deutsches Institut für Wirtschaftsforschung.

Bach, Stefan, und Thomas Mertz. 2016. Vor der Erbschaftsteuerreform: Nutzung der Firmenprivilegien hat Minderjährige zu Multimillionären gemacht. DIW-Wochenbericht 83:812-820.

Bach, Stefan, und Andreas Thiemann. 2016. Reviving Germany's wealth tax creates high revenue potential. DIW Economic Bulletin 6(4):50-59.

Bach, Stefan, Andreas Thiemann, und Aline Zucco. 2019. Looking for the missing rich: Tracing the top tail of the wealth distribution. International Tax and Public Finance 26(6):1234-1258.

Bank, Julian. 2015. Die Piketty-Rezeption in Deutschland. In Thomas Piketty und die Verteilungsfrage, Hrsg. Peter Bofinger, Gustav A. Horn, Kai D. Schmid, und Till van Treeck, 9-36. Leipzig: SE.

Bank, Julian. 2017. Economic inequality in the German quality press: framing concerns about inequality and redistribution. International Journal of Communication 11:4370-4395.

Barnes, Lucy. 2021. Taxing the rich: public preferences and public understanding. Journal of European Public Policy https://doi.org/10.1080/13501763.2021.1992485.

Beckert, Jens. 2013. Erben in der Leistungsgesellschaft. Frankfurt a.M.: Campus.

Beckert, Jens, und Lukas R. Arndt. 2017. Verdient-unverdient. Der öffentliche Diskurs um die Erbschaftssteuer in Deutschland und Österreich. Berliner Journal für Soziologie 27(2):271-291.

Benson, Rodney. 2016. Institutional forms of media ownership and their modes of power. In The journalistic institution reexamined: digital challenges and professional reorientations, Hrsg. Martin Eide, Leif Ove Larsen, und Helle Sjøvaag, 27-48. Bistol, Chicago: Intellect.

Benson, Rodney. 2019. Rethinking the sociology of media ownership. In Routledge handbook of cultural sociology, 2. Aufl., Hrsg. Laura Grindstaff, Ming-Cheng M. Lo, und John R. Hall, 387-396. London: Routledge.

Bergmann, Knut, und Leonhard Novy. 2012. Chancen und Grenzen philanthropischer Finanzierungsmodelle. Aus Politik und Zeitgeschichte 62(29-31):33-39.

Burkhardt, Kai. 2012. Frankfurter Allgemeine Zeitung. Mediendatenbank. https://www.mediadb.eu/forum/ zeitungsportraets/faz.html. Zugegriffen: 24. Juni 2020.

Castells, Manuel. 2009. Communication power. New York: Oxford University Press.

Clift, Ben, und Sean McDaniel. 2017. Is this crisis of French socialism different? Hollande, the rise of Macron, and the reconfiguration of the left in the 2017 presidential and parliamentary elections. Modern \& Contemporary France 25(4):403-415.

Destatis. 2018. Öffentliche Finanzen, Steuern, Personal. https://www-genesis.destatis.de/genesis/online/ data. Zugegriffen: 24. Juni 2020. 
Drometer, Marcus, Marco Frank, Maria Pérez Hofbauer, Carla Rhode, Sebastian Schworm, und Tanja Stitteneder. 2018. Wealth and inheritance taxation: an overview and country comparison. ifo DICE Report 16(2):45-54.

Elsässer, Lea, Svenja Hense, und Armin Schäfer. 2017. „Dem Deutschen Volke“? Die ungleiche Responsivität des Bundestags. Zeitschrift für Politikwissenschaft 27:161-180.

Epp, Derek A., und Jay T. Jennings. 2020. Inequality, media frames, and public support for welfare. Public Opinion Quarterly 84(3):629-653.

Fastenrath, Florian, Paul Marx, Achim Truger, und Helena Vitt. 2021. Why is it so difficult to tax the rich? Evidence from German policy-makers. Journal of European Public Policy https://doi.org/10.1080/ 13501763.2021.1992484.

Ferschli, B, D. Grabner, und H. Theine. 2019. Zur Politischen Ökonomie der Medien inDeutschland: Eine Analyse der Konzentrationstendenzen und Besitzverhältnisse. Institut sozial-ökologische Wirtschaftsforschung report 118, München.

Fessler, Pirmin, und Martin Schürz. 2018. Private wealth across European countries: the role of income, inheritance and the welfare state. Journal of Human Development and Capabilities 19:521-549.

Franke, Benedikt, Dirk Simons, und Dennis Voeller. 2016. Who benefits from the preferential treatment of business property under the German inheritance tax? Journal of Business Economics 86(9):997-1041.

Gehrs, Oliver. 2005. Der Spiegel-Komplex. Wie Stefan Aust das Blatt für sich wendete. München: Droemer.

Grabka, Markus. 2014. Verteilung und Struktur des Reichtums in Deutschland. In Reichtum, Philanthropie und Zivilgesellschaft, Hrsg. Wolfgang Lauterbach, Michael Hartmann, und Miriam Ströing, 21-45. Wiesbaden: Springer.

Grisold, Andrea. 2004. Kulturindustrie Fernsehen: Zum Wechselverhältnis von Ökonomie und Massenmedien. Wien: Löcker.

Grisold, Andrea. 2009. Zur Politischen Ökonomie der Medien. Eine heterodoxe Erweiterung. In Heterodoxe Ökonomie, Hrsg. Joachim Becker, Andrea Grisold, Gertraude Mikl-Horke, Reinhard Pirker, Hermann Rauchenschwandtner, Oliver Schwank, Elisabeth Springler, und Engelbert Stockhammer, 147-176. Marburg: Metropolis.

Grisold, Andrea, und Paschal Preston (Hrsg.). 2020. Economic inequality and news media: discourse, power, and redistribution. Oxford: Oxford University Press. https://doi.org/10.1093/oso/9780190053 901.001.0001.

Grisold, Andrea, und Hendrik Theine. 2017. How come we know? The media coverage of economic inequality. International Journal of Communication 11:4265-4284.

Grisold, Andrea, und Hendrik Theine. 2018. Zur Vermittlungsrolle von Massenmedien am Thema „Ungleichheit“. Die Piketty-Rezeption. Wirtschaft und Gesellschaft 44(2):191-218.

Grisold, Andrea, und Hendrik Theine. 2020. "Now, what exactly is the problem?" On the media coverage of economic inequalities and redistribution policies-the Piketty case. Journal of Economic Issues 54(4):1071-1094. https://doi.org/10.1080/00213624.2020.1829905.

Groll, Tina. 2012. die tageszeitung (taz). Mediendatenbank. https://www.mediadb.eu/forum/zeitungsportra ets/die-tageszeitung-taz.html. Zugegriffen: 24. Juni 2020.

Haider, Ahmad. 2017. Frequency Analysis as a way of uncovering news foci: Evidence from the Guardian and the New York Times. International Journal of English Linguistics 7(2):1-20.

Herman, Edward S., und Noam Chomsky. 2002. Manufacturing consent. The political economy of the mass media. New York: Pantheon.

Houben, Henriette, und Ralf Maiterth. 2011. Erbschaftsteuer und Erbschaftsteuerreform in Deutschland: eine Bestandsaufnahme. Vierteljahrshefte zur Wirtschaftsforschung 80(4):161-188.

van Kommer, Victor, und Lambert Kosters. 2013. Möglichkeiten der Wiedereinführung der Vermögensteuer in Deutschland und denkbare Alternativen. Kurzgutachten zu Optionen einer Reform der Vermögensteuer in Deutschland. https://www.boeckler.de/data/wsi_kurzgutachten_kommer_kosters.pdf. Zugegriffen: 10. März 2021. erstellt im Auftrag des Wirtschafts- und Sozialwissenschaftlichen Instituts (WSI) in der Hans-Böckler-Stiftung, Utrecht.

Kommission zur Ermittlung der Konzentration im Medienbereich (kek). 2019. Mediendatenbank. https:// www.kek-online.de/medienkonzentration/mediendatenbank\#/. Zugegriffen: 24. Juni 2020.

Krüger, Uwe. 2019. Meinungsmacht. Der Einfluss von Eliten auf Leitmedien und Alpha-Journalisten eine kritische Netzwerkanalyse, 2. Aufl., Köln: Herbert von Halem.

Krüger, Uwe, Holger Pötzsch, und Hendrik Theine. 2021. Wie neoliberal sind die Medien? In Zerreiß 3 proben: Leitmedien, Liberalismus und Liberalität, Hrsg. Stephan Russ-Mohl, Christian Hoffmann, 113-125. Köln: Herbert von Harlem. 
Krugman, Paul. 2017. Why we're in a new gilded age. In After Piketty-The agenda for economics and inequality, Hrsg. Heather Boushey, J. Bradford DeLong, und Marshall Steinbaum, 60-71. Cambridge: Harvard University Press.

Lehrer, Mark, und Stefan Schmid. 2015. Germany's industrial family firms: prospering islands of social capital in a financialized world? Competition \& Change 19(4):301-316.

Leitner, Sebastian. 2016. Drivers of wealth inequality in euro area countries: the effect of inheritance and gifts on household gross and net wealth distribution analysed by applying the Shapley value approach to decomposition. European Journal of Economics and Economic Policies: Intervention 13(1):114-136.

Lemke, Matthias, und Gregor Wiedemann (Hrsg.). 2016. Text Mining in den Sozialwissenschaften: Grundlagen und Anwendungen zwischen qualitativer und quantitativer Diskursanalyse. Wiesbaden: Springer.

Lichtenstein, Dennis, Markus Rhomberg, und Michaela Böhme. 2016. Konsonant oder interessengeleitet? Eine Frame-Analyse zur Berichterstattung über die Vermögensteuer im Wahljahr 2013. In Understanding Inequality: Social Costs and Benefits, Hrsg. A. Machin, N. Stehr, 325-345. Wiesbaden: Springer.

Maiterth, Ralf. 2013. Gefährdung deutscher Unternehmen durch die Erbschaftsteuer? - Eine empirische Analyse. In Daten in der wirtschaftswissenschaftlichen Forschung, Hrsg. Dierk Hirschel, Peter Paic, und Markus Zwick, 135-171. Wiesbaden: Springer.

Mautner, Gerlinde. 2015. Checks and Balances: How corpus linguistics can contribute to CDA. In Methods of critical discourse studies, Hrsg. Ruth Wodak, Michael Meyer, 154-179. London: SAGE.

Mosco, Vincent. 2009. The political economy of communication. Los Angeles: SAGE.

Neimanns, Erik. 2021. Welfare states, media ownership and attitudes towards redistribution. Journal of European Public Policy https://doi.org/10.1080/13501763.2021.1986117.

Nullmeier, Frank, Dominika Biegon, Jennifer Gronau, Sebastian Haunss, Falk Lenke, Henning Schmidtke, und Steffen Schneider. 2014. Marktwirtschaft in der Legitimationskrise? Ein internationaler Vergleich. Frankfurt a.M.: Campus.

Petring, Alexander. 2016. Die drei Welten des Gerechtigkeitsjournalismus? Text Mining in FAZ, taz und SZ zu sozialer Gerechtigkeit und Ungleichheit. In Text Mining in den Sozialwissenschaften. Grundlagen und Anwendungen zwischen qualitativer und quantitativer Diskursanalyse, Hrsg. Matthias Lemke, Gregor Wiedemann, 369-393. Wiesbaden: Springer.

Piketty, Thomas. 2011. On the long-run evolution of inheritance: France 1820-2050. The Quarterly Journal of Economics 126(3):1071-1131.

Piketty, Thomas. 2014. Capital in the twenty-first century. Oxford: Cambridge University Press.

Piketty, Thomas, und Gabriel Zucman. 2015. Wealth and inheritance in the long run. In Handbook of income distribution, Bd. 2A, Hrsg. Anthony Atkinson, François Bourguignon, 1303-1368. Amsterdam: Elsevier.

Pointner, Nicola. 2010. In den Fängen der Ökonomie? Ein kritischer Blick auf die Berichterstattung über Medienunternehmen in der deutschen Tagespresse. Wiesbaden: Springer VS.

Rehm, Miriam, und Matthias Schnetzer. 2015. Property and Power: Lessons from Piketty and New Insights from the HFCS. European Journal of Economics and Economic Policies: Intervention 12(2):204-219.

Rieder, Maria, und Hendrik Theine. 2019. 'Piketty is a genius, but...': an analysis of journalistic delegitimation of Thomas Piketty's economic policy proposals. Critical Discourse Studies 16(3):248-263. https://doi.org/10.1080/17405904.2019.1573148.

Roine, Jesper, und Daniel Waldestrom. 2009. Wealth concentration over the path of development: Sweden 1873-2006. Scandinavian Journal of Economics 111(1):151-187.

Röper, Horst. 2018. Zeitungsmarkt 2018: Pressekonzentration steigt rasant. Media Perspektiven 5:216-234.

Sachverständigenrat zur Begutachtung der gesamtwirtschaftlichen Entwicklung. 2019. Jahresgutachten 2019/20: Den Strukturwandel meistern. https://www.sachverstaendigenrat-wirtschaft.de/jahresgutac hten-2019.html. Zugegriffen: 24. Juni 2020.

Sasse, Saskia. 2012. Die Welt. Mediendatenbank. https://www.mediadb.eu/forum/zeitungsportraets/diewelt.html. Zugegriffen: 10. Juni 2020.

Scheve, Kenneth, und Daniel Stasavage. 2016. Taxing the rich: A history of fiscal fairness in the United States and Europe. Princeton: Princeton University Press.

Scholz, Birger, und Achim Truger. 2016. Erbschaftsteuer-Reform 2016: Eine Aktualisierung der Fallbeispiele nach dem Kompromiss im Vermittlungsausschuss. Kurzexpertise im Auftrag von Campact. https://blog.campact.de/wp-content/uploads/2016/10/Erbschaftsteuer_Fallbeispiele_Campact_ Scholz_Truger_11.10.2016.pdf. Zugegriffen: 24. Juni 2020. 
Schröder, Martin, und Florian Vietze. 2015. Mediendebatten über soziale Ungleichheit, Armut und soziale Gerechtigkeit seit 1946 und wie sie mit Einkommensungleichheit zusammenhängen. Zeitschrift für Soziologie 44(1):42-62.

Schulz, Klaus Dieter. 1986. Unternehmerinteresse und Wirtschaftssystem. Beiträge der Unternehmer zur politischen Entwicklung der Bundesrepublik Deutschland. Frankfurt a.M.: Haag + Herchen.

Schulz, Winfried. 1989. Massenmedien und Realität. Die ,ptolemäische " und die ,kopernikanische " Auffassung. In Massenkommunikation. Theorien, Methoden, Befunde, Hrsg. Max Kaase, Winfried Schulz, 135-149. Opladen: Westdeutscher Verlag.

Silge, Julia, und David Robinson. 2017. Text mining with R: a tidy approach. Sebastopol: O'Reilly.

Smith Ochoa, Christopher. 2020. Trivializing inequality by narrating facts: a discourse analysis of contending storylines in Germany. Critical Policy Studies 14(3):319-338.

Stiglitz, Joseph. 2019. People, power, and profits: Progressive capitalism for an age of discontent. New York: Norton.

Straka, Milan. 2018. UDPipe 2.0 prototype at CoNLL 2018 UD shared task. In Proceedings of CoNLL 2018: The SIGNLL Conference on Computational Natural Language Learning, 197-207. Stroudsburg: Association for Computational Linguistics.

Subtirelu, Nicolas, und Paul Baker. 2017. Corpus-based approaches. In The Routledge Handbook of Critical Discourse Studies, Hrsg. J. Flowerdew, und J. Richardson, 106-119. London: Routledge.

Süddeutsche Zeitung. 2013. Erste Umarmung unter Landsleuten. Süddeutsche Zeitung. https://www. sueddeutsche.de/panorama/neu-russe-depardieu-trifft-putin-erste-umarmung-unter-landsleuten- 1 . 1565760 (Erstellt: 6. Jan. 2013). Zugegriffen: 24. Juni 2020.

Theine, Hendrik. 2020. Reichtum und Macht. Eine politökonomische Perspektive auf die Erbschaftsbesteuerung in Deutschland. In Ökonomie und Gesellschaft (Jahrbuch 31). Ökonomie in der Krise: Analyse - Kritik - Umgestaltung, Hrsg. Gerd Grötzinger, Arne Heise, und Helge Peukert, 79-96. Marburg: Metropolis.

Theine, Hendrik. 2021. Economists in public discourses: The case of wealth and inheritance taxation in the German press. In Power and influence of economists contributions to the social studies of economics, Hrsg. Jens Maesse, Stephan Pühringer, Thierry Rossier, und Pierre Benz, 188-206. London: Routledge.

Theine, Hendrik, und Daniel Grabner. 2020. Trends in economic inequality and news mediascape. In Economic inequality and news media: discourse, power, and redistribution, Hrsg. Andrea Grisold, Paschal Preston, 21-47. Oxford: Oxford University Press. https://doi.org/10.1093/oso/9780190053901. 003.0002 .

Theine, Hendrik, und Andrea Grisold. 2020. Streitfall Vermögenssteuer: Defizite in der Medienberichterstattung. OBS-Arbeitspapier, Bd. 43. Frankfurt a.M.: Otto Brenner Stiftung.

Theine, Hendrik, und Maria Rieder. 2019. The billionaires boot boys start screaming — A critical analysis of economic policy discourses in reaction to Piketty's 'capital in the 21 st century. In Critical policy discourse analysis, Hrsg. Nicolina Montesano Montessori, Michael Farrelly, und Jane Mulderrig, 169-192. Cheltenham: Edward Elgar. https://doi.org/10.4337/9781788974967.00013.

Theine, Hendrik, und Mario Tatschwer. 2021. Ungleichheit: Warum wir uns die Reichen nicht mehr leisten können. In Klimasoziale Politik: eine gerechte und emissionsfreie Gesellschaft gestalten, Hrsg. Armutskonferenz, attac und Beigewum, 119-129. Wien: Bahoe Books.

Volkmann, Ute. 2006. Legitime Ungleichheiten. Journalistische Deutungen vom „sozialdemokratischen Konsensus“ zum „Neoliberalismus“. Wiesbaden: VS.

Weischenberg, Siegfried, Maja Malik, und Armin Scholl. 2005. Journalismus in Deutschland 2005. Media Perspektiven 7:346-361.

Wieland, Joachim. 2003. Rechtliche Rahmenbedingungen für die Wiedereinführung der Vermögensteuer. Rechtsgutachten erstattet für ver.di.

Wolter, Philipp. 2016. Neoliberale Denkfiguren in der Presse. Wie ein Wirtschaftskonzept die Meinungshoheit erobert. Marburg: Metropolis. 